\title{
Large Scale Anisotropic Bias from Primordial non-Gaussianity
}

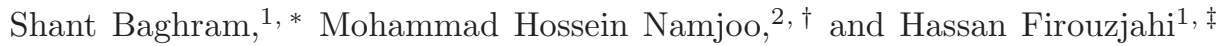 \\ ${ }^{1}$ School of Astronomy, Institute for Research in Fundamental Sciences (IPM), P. O. Box 19395-5531, Tehran, Iran \\ ${ }^{2}$ School of Physics, Institute for Research in Fundamental Sciences (IPM), P. O. Box 19395-5531, Tehran, Iran
}

\begin{abstract}
In this work we study the large scale structure bias in models of anisotropic inflation. We use the Peak Background Splitting method in Excursion Set Theory to find the scale-dependent bias. We show that the amplitude of the bias is modified by a direction-dependent factor. In the specific anisotropic inflation model which we study, the scale-dependent bias vanishes at leading order when the long wavelength mode in squeezed limit is aligned with the anisotropic direction in the sky. We also extend the scale-dependent bias formulation to the general situations with primordial anisotropy. We find some selection rules indicating that some specific parts of a generic anisotropic bispectrum is picked up by the bias parameter. We argue that the anisotropic bias is mainly sourced by the angle between the anisotropic direction and the long wavelength mode in the squeezed limit.
\end{abstract}

\section{INTRODUCTION}

Inflation [1] has emerged as the leading paradigm for the theory of early Universe and structure formation. Basic predictions of inflation indicate that the curvature perturbations are nearly scale-invariant, nearly adiabatic and nearly Gaussian which are in very good agreements with cosmological observations such as WMAP [2] and PLANCK [3]. The simplest models of inflation are based on a scalar field rolling slowly on a flat potential. Any detection of primordial non-Gaussianity (NG) will have significant implications for inflationary model buildings, for a review see [4 6 ]. For example, many models of single field inflation predict a very small amount of local non-Gaussianity in the squeezed limit $f_{N L} \sim\left(1-n_{s}\right)$ 7], in which $n_{s}$ is the curvature perturbation power spectrum spectral index and $f_{N L}$ parametrizes the amplitude of local NG. With $n_{s} \simeq 0.96$ from PLANCK [3], one expects $f_{N L} \sim \mathcal{O}\left(10^{-2}\right)$ for conventional models of single field inflation. However, this expectation is violated if the system has not reached the attractor regime [8, 9$]$ or if one allows for a non Bunch-Davies initial condition [10 13]. Furthermore, inflationary models with large NG predicts different shapes for bispectrum. Therefore, any detection or otherwise of large primordial NG with different shapes will go a long way to rule out many inflationary scenarios or put constraints on model parameters. Having this said, the recent PLANCK collaboration data [14] showed no significant deviation from Gaussian initial conditions. PLANCK constrained the amplitude of NG for different known shapes and accordingly the Gaussian initial conditions are consistent with the picture.

The most suitable cosmological observation to constrain the primordial NG is CMB. This is because the perturbations in the last scattering surface are in the linear regime and the fingerprints of non-Gaussianity are mainly preserved 15, 16]. However, recently the interests in Large Scale Structure (LSS) observations and their implications for non-Gaussianity are boosted due to the theoretical findings of scale-dependent bias [17]. In general, the distribution of baryonic matter in the Universe, mainly clustered in galaxies and the clusters of galaxies, is the fundamental observable of LSS [18, 19]. The distribution of galaxies and clusters of galaxies can be studied by a): the mass function of the structures (i.e. galaxies and cluster of galaxies) and b): the correlation functions, power spectrum and even higher moments of distribution. In the standard theories of structure formation, the Gaussianity assumption plays a crucial role in finding the distribution of structures via the primordial density contrast distribution [20]. Accordingly, changing the initial condition from a Gaussian to non-Gaussian primordial density perturbations will change the mass function of structures. This change mainly shows itself in the tail of distribution function. Consequently, this effect manifests itself mainly in the statistics of the clusters of galaxies in high mass and high redshifts distributions 21 25]. The bispectrum of LSS observations is also affected by the primordial NG and by the secondary NG induced by the non-linear growth of structures [26]. The NG introduced by non-linear growth of structures has its own signatures on the bispectrum of structures, but its shape changes in deep non-linear regime. One can find the bispectrum of galaxies and clusters of galaxies to detect the primordial non-Gaussianity and distinguish its effect from the effects

\footnotetext{
*Electronic address: baghram-AT-ipm.ir

${ }^{\dagger}$ Electronic address: mh.namjoo-AT-ipm.ir

$\ddagger$ Electronic address: firouz-AT-ipm.ir
} 
of gravitational instability. Recent works have shown that the galaxy bispectrum can be a very promising way to constrain primordial NG [18, 27 29]. On the other hand, the primordial NG may have an effect on the clustering of halos. As an intuitive example we can consider the local NG where the long wavelength mode changes the background linear density fluctuations. This change, in the picture of linear bias theory, will have an influence on the density peaks where structures are formed. In other words, the non-Gaussian long mode changes the threshold which structure goes from linear to non-linear regime.

The primordial NG has unique feature in LSS by introducing a scale-dependent bias [17, 30, 31]. This fingerprint of primordial NG provides the opportunity to constrain primordial NG using the power spectrum of galaxies. Many works are done in numerical simulations to check the scale-dependence of the bias parameter introduced by primordial NG and also to study the change in the statistics of structures in Universe [25, 32, 33]. From the observational side different groups used the LSS probes to constrain the primordial NG [34 36]. Furthermore, there are other LSS observables such as the Integrated Sachs Wolfe cross correlation with the galaxy power spectrum [36], the 3D bispectrum of Ly-alpha forest, the redshifted 21-cm signal from the post re-ionization epoch [37, 38], the statistics of voids [39], the cosmological weak lensing [40] etc. which can also be used to study the primordial NG. In a word, the LSS observations will become a very important tool, complementary to CMB observations, to constrain the properties of primordial NG.

There have been some indications of statistical anisotropies on CMB power spectrum. Although The statistical significance of the violation of statistical isotropy is not high in WMAP data [41, 42], but nonetheless the possibility of having statistically anisotropic seed perturbations are intriguing. Recently the data from PLANCK collaboration also confirmed the anomalies observed by WMAP, including the anisotropy in CMB sky [43]. This observation triggers the interests in anisotropic models both theoretically and observationally.

The statistical anisotropy is usually parameterized via [4] $\mathcal{P}_{\zeta}=\mathcal{P}_{\zeta_{0}}\left(1+g_{*}(\hat{k} . \hat{n})^{2}\right)$ in which $\hat{n}$ is the preferred direction in sky, $\mathcal{P}_{\zeta}$ is the curvature perturbation power spectrum of the Fourier mode $\vec{k}$ with the direction along the unit vector $\hat{k}$ and $\mathcal{P}_{\zeta_{0}}$ represents the isotropic power spectrum. Constraints from CMB and large scale structure indicate that $\left|g_{*}\right| \lesssim 0.4[14,45,46]$. Recently the bispectrum and the trispectrum in a model of anisotropic inflation [47] have been calculated in [48 51]. It has been shown that large non-Gaussianities with non-trivial shapes are generated. Considering our motivation in using non-Gaussianity fingerprints in LSS as a probe of inflationary universe, we would like to study the effects of large scale-dependent and orientation-dependent bispectrum on bias in these models.

The rest of the paper is organized as follows: In Section II we present the anisotropic inflation model and the corresponding bispectrum which is used in subsequent analysis. In Section II we review the basics of halo bias with non-Gaussian initial conditions. In Section IV we present a general mathematical formulation for the anisotropic bispectrum in terms of spherical harmonics which can be used to calculate the halo bias in models with generic anisotropic bispectrum. In Section $\nabla$ we present our results of halo bias in the models of anisotropic inflation with the anisotropic bispectrum obtained in Section We leave some technical issues of halo bias analysis into the Appendix.

In this paper we work with the natural unit in which $c=\hbar=1$.

\section{ANISOTROPIC INFLATIONARY MODEL}

In this section we review the anisotropic inflation model and its anisotropic bispectrum which will be used in subsequent analysis.

The best method to introduce statistical anisotropies is to incorporate $U(1)$ gauge fields or vector fields in models of inflation. However, due to conformal invariance of the standard Maxwell theory in an expanding background, the background gauge field and its quantum fluctuations are diluted during inflation. Therefore, in order to produce an almost scale-invariant power spectrum of gauge field fluctuations, one has to consider a time-dependent gauge kinetic coupling. This prescription was originally used in 52, 53] in the context of primordial magnetic field. An interesting model of anisotropic inflation is introduced in [47] in which it is shown that, with a suitably chosen gauge kinetic coupling, the inflationary system has an attractor solution in which the gauge field energy density reaches a small but observationally detectable fraction of the total energy density. As a result, the anisotropy produced are at the order of slow-roll parameters.

The Lagrangian of the system is

$$
S=\int d^{4} x \sqrt{-g}\left[\frac{M_{P}^{2}}{2} R-\frac{1}{2} \partial_{\mu} \phi \partial^{\mu} \phi-\frac{f^{2}(\phi)}{4} F_{\mu \nu} F^{\mu \nu}-V(\phi)\right],
$$

in which $\phi$ is the inflaton field and $F_{\mu \nu}=\partial_{\mu} A_{\nu}-\partial_{\nu} A_{\mu}$ is the field strength associated with the $U(1)$ gauge field $A_{\mu}$. 
The background is in the form of Bianchi I universe with the metric

$$
\begin{aligned}
d s^{2} & =-d t^{2}+e^{2 \alpha(t)}\left(e^{-4 \sigma(t)} d x^{2}+e^{2 \sigma(t)}\left(d y^{2}+d z^{2}\right)\right) \\
& =-d t^{2}+a(t)^{2} d x^{2}+b(t)^{2}\left(d y^{2}+d z^{2}\right) .
\end{aligned}
$$

Here $H \equiv \dot{\alpha}$ is interpreted as the average Hubble expansion rate, $H_{a} \equiv \dot{a} / a$ and $H_{b} \equiv \dot{b} / b$ are the expansion rates along the spatial directions $x$ and $y$ and $\dot{\sigma} / H \equiv\left(H_{b}-H_{a}\right) / H$ is a measure of anisotropic expansion. We note that this metric enjoys only a two-dimensional rotational symmetry in $y-z$ plane.

The details of the dynamics of the system are given in [47, 50]. For the simple chaotic potential $V=m^{2} \phi^{2} / 2$, the conformal coupling (the time-dependent gauge kinetic coupling) is chosen to be

$$
f(\phi)=\exp \left(\frac{c \phi^{2}}{2 M_{P}^{2}}\right)
$$

where $c$ is a constant. With $c \simeq 1$, one can check that the system admits the attractor solution in which the ratio of the gauge field energy density in the form of electric field energy density is a small and constant fraction of the total energy density. Defining the fraction of electric field energy density to the potential energy as

$$
R \equiv \frac{\dot{A}^{2} f(\phi)^{2} e^{-2 \alpha}}{2 V}
$$

during the attractor regime one obtains

$$
R=\frac{c-1}{2 c} \epsilon_{H}=\frac{I}{2} \epsilon_{H}
$$

in which $\epsilon_{H} \equiv-\dot{H} / H^{2}$ is the slow-roll parameter and $I \equiv \frac{c-1}{c}$.

The power spectrum of the curvature perturbation is defined via

$$
\left\langle\zeta_{\mathbf{k}} \zeta_{\mathbf{k}^{\prime}}\right\rangle=(2 \pi)^{3} P_{\zeta}(\mathbf{k}) \delta^{3}\left(\mathbf{k}+\mathbf{k}^{\prime}\right) \quad, \quad \mathcal{P}_{\zeta} \equiv \frac{k^{3}}{2 \pi^{2}} P_{\zeta}(\mathbf{k})
$$

For the particular anisotropic inflation model described above the power spectrum was calculated in [48 51, 54 59] which has the form

$$
P_{\zeta}(\vec{k})=P_{0}\left(1+g_{*}(\hat{k} . \hat{n})^{2}\right)
$$

where the anisotropy parameter $g_{*}$ is given by

$$
g_{*} \equiv-24 I N\left(k_{1}\right) N\left(k_{2}\right) .
$$

Here $N\left(k_{i}\right)$ represents the number of e-folding that the mode of interest $k$ leaves the horizon. In our notation, the number of e-folding is counted backwards from the time of the end of inflation by $a(N)=a_{f} \exp (N)$ so $N \leq 0$. For example, $N=N_{C M B}=-60$ to solve the flatness and the horizon problem. As a result, $N(k)$ is calculated to be

$$
N(k)-N_{C M B}=\ln \left(\frac{k}{k_{C M B}}\right),
$$

in which $k_{C M B}$ represents the comoving mode which leaves the horizon at $N_{C M B}=-60$ e-folds before the end of inflation. To satisfy the observational constraints from CMB and large scale structure we require $\left|g_{*}\right|<0.3$ [14, 45, 46] corresponding to $I \lesssim 10^{-5}[55[58]$.

The bispectrum of curvature perturbations, $B_{\zeta}\left(\vec{k}_{1}, \vec{k}_{2}, \vec{k}_{3}\right)$, is defined via

$$
\left\langle\zeta\left(\vec{k}_{1}\right) \zeta\left(\vec{k}_{2}\right) \zeta\left(\vec{k}_{3}\right)\right\rangle \equiv(2 \pi)^{3} \delta^{3}\left(\vec{k}_{1}+\vec{k}_{2}+\vec{k}_{3}\right) B_{\zeta}\left(\vec{k}_{1}, \vec{k}_{2}, \vec{k}_{3}\right) .
$$

The Bispectrum for the model of anisotropic inflation was calculated using in-in formalism in [48, 49] and using $\delta N$ formalism in [50] with the result

$$
B_{\zeta}\left(\vec{k}_{1}, \vec{k}_{2}, \vec{k}_{3}\right)=288 I N\left(k_{1}\right) N\left(k_{2}\right) N\left(k_{3}\right)\left(C\left(\vec{k}_{1}, \vec{k}_{2}\right) P_{0}\left(k_{1}\right) P_{0}\left(k_{2}\right)+2 \text { perm. }\right) .
$$


Here the anisotropic shape function $C\left(\vec{k}_{1}, \vec{k}_{2}\right)$ is defined as:

$$
C\left(\vec{k}_{1}, \vec{k}_{2}\right) \equiv\left(1-\left(\widehat{k}_{1} \cdot \widehat{n}\right)^{2}-\left(\widehat{k}_{2} \cdot \widehat{n}\right)^{2}+\left(\widehat{k}_{1} \cdot \widehat{n}\right)\left(\widehat{k}_{2} \cdot \widehat{n}\right)\left(\widehat{k}_{1} \cdot \widehat{k}_{2}\right)\right)
$$

where $\hat{n}$ is the specific anisotropic direction in the sky. Note that in Eq. (11), $P_{0}\left(k_{i}\right)$ represents the isotropic power spectrum so all anisotropies are encoded in shape function $C\left(\vec{k}_{1}, \vec{k}_{2}\right)$ (and the appropriate permutations) with the amplitude $288 I N\left(k_{1}\right) N\left(k_{2}\right) N\left(k_{3}\right)$.

It is instructive to look into the bispectrum in the squeezed limit in which one mode is much longer than the other two, say $k_{3} \ll k_{1} \simeq k_{2}$, so from the condition $\sum_{i} \vec{k}_{i}=0$ we also conclude that $\vec{k}_{1} \simeq-\vec{k}_{2}$. In this limit, one obtains

$$
B_{\zeta}\left(k_{1}, k_{2}, k_{3}\right) \simeq 24 P_{0}\left(k_{1}\right) P_{0}\left(k_{3}\right)\left|g_{*}\left(k_{1}\right)\right| N\left(k_{3}\right) \times\left[1-\left(\hat{k}_{1} \cdot \hat{n}\right)^{2}-\left(\hat{k}_{3} \cdot \hat{n}\right)^{2}+\left(\hat{k}_{1} \cdot \hat{n}\right)\left(\hat{k}_{3} \cdot \hat{n}\right)\left(\hat{k}_{1} \cdot \hat{k}_{3}\right)\right] \quad\left(k_{3} \ll k_{1} \simeq k_{2}\right)
$$

in which to obtain the above result, Eq. (8) has been used to express the parameter $I N\left(k_{1}\right) N\left(k_{2}\right)$ in terms of $g_{*}$.

The non-Gaussianity parameter $f_{N L}$ is defined in the squeezed limit $k_{3} \ll k_{1} \simeq k_{2}$ via [4, 5]

$$
f_{N L}\left(\vec{k}_{1}, \vec{k}_{2}, \vec{k}_{3}\right)=\lim _{k_{3} \rightarrow 0} \frac{5}{12} \frac{B_{\zeta}\left(\vec{k}_{1}, \vec{k}_{2}, \vec{k}_{3}\right)}{P_{\zeta}\left(k_{1}\right) P_{\zeta}\left(k_{3}\right)} .
$$

In general, $f_{N L}$ is an orientation-dependent and scale-dependent quantity. As an order of magnitude estimation, and neglecting the logarithmic scale-dependence in $N\left(k_{i}\right)$, we can define an orientation-dependent effective $f_{N L}^{e f f}$ via

$$
f_{N L}^{e f f}=240 I N_{C M B}^{3} C\left(\vec{k}_{1}, \vec{k}_{2}\right)
$$

keeping in mind that $N(k)=N_{C M B}+\ln \left(k / k_{C M B}\right)$. Setting $N_{C M B}=60$, we can easily get $f_{N L}^{e f f} \sim 60$ with $g_{*} \sim 0.1$, compatible with observational constraints.

A very interesting observation from Eq.(13) is that when the long wavelength mode $\vec{k}_{3}$ is in the direction of anisotropy, (i.e. $\vec{k}_{3} \| \hat{n}$ ), then the term inside the big-bracket in Eq.(13) vanishes. Consequently, in this configuration, we do not expect to see the NG effects in LSS. We discuss this feature in more details in Sec III

For the subsequent analysis we adopt the coordinate system such as the anisotropic direction $\hat{n}$ coincides with the $\hat{z}$ direction in the spherical coordinates so the other momentum vectors are described by $\hat{k}_{1}=\left(\theta_{1}, \psi_{1}\right)$ and $\hat{k}_{2}=\left(\theta_{2}, \psi_{2}\right)$, where $\theta$ and $\psi$ are the polar and azimuthal angles in spherical coordinates, respectively. For the future reference, we also need the angles between two arbitrary unit vectors $\hat{q}_{i}=\left(\theta_{q_{i}}, \psi_{q_{i}}\right)$ defined via $\cos \gamma=\hat{q}_{1} . \hat{q}_{2}$, which is

$$
\cos \gamma=\sin \left(\theta_{q_{1}}\right) \sin \left(\theta_{q_{2}}\right) \cos \left(\psi_{q_{1}}-\psi_{q_{2}}\right)+\cos \theta_{q_{1}} \cos \theta_{q_{2}}
$$

The power spectrum and the bi-spectrum presented in Eqs. (7) and (11) are for the particular model of anisotropic inflation as studied in [48 50]. For a generic anisotropic model the most general power spectrum can be written as 60 .

$$
P(\vec{k})=P_{0}(k)\left[1+\sum_{L M} g_{L M}(k) Y_{L M}(\hat{k})\right]
$$

where $P_{0}$ is the isotropic power spectrum, $Y_{L M}(\hat{k})$ (with $L \geq 2$ ) are spherical harmonics and $g_{L M}(k)$ quantify the departure from statistical isotropy as a function of wavenumber $k$. Since each Fourier mode $\vec{k}$ is related to $-\vec{k}$, in the case of real $g_{L M}(k)$, the multipole moment $L$ must be even, and in the limit of $k \rightarrow 0$ we recover the isotropic power spectrum $P_{0}(k)$. However, in the general case, (real and imaginary $g_{L M}$ ), we have

$$
g_{L M}^{*}=(-1)^{L} g_{L-M}
$$

This condition is imposed by the fact that the matter power spectrum is a real quantity.

Comparing Eq. (17) with Eq. (77) for our particular anisotropic inflation model we have $g_{20} \propto g_{*}$ while the rest of $g_{L M}$ are zero. In Section [V] we extend the general definition of Eq. (17) for the power spectrum to the bispectrum and look into its implications in halo bias analysis. 


\section{BIAS}

In this section we review the concept of bias, a parameter that shows the dependence of dark matter halo abundance to the background dark matter density perturbations. The reader who is familiar with these analysis can directly jump to the next Sections in which we present our results of halo bias for anisotropic primordial power spectrum and bispectrum. It is worth to mention that in this work we are not interested in galaxy bias, which is the weighted integral of the halo bias, corresponding to the mechanism of halo occupation distribution (HOD).

In order to find an expression for the bias parameter we follow the work by Scoccimarro et al 61. However, there are other studies which use Excursion Set Theory (EST) to calculate the halo bias. In Adshead et al [62] the authors solved the more complicated problem of non-spherical halos for which the collapse threshold becomes scale-dependent. In D'Aloisio et al. 63] EST is extended to path integral approach taking into account the non-Markovianity effects of random walks in EST.

The halo bias relates the halo abundance to the dark matter over-density. In Excursion Set Theory (EST) [64] it is defined as

$$
b(k, z)=\frac{\delta_{h}}{\delta_{m}}
$$

where $\delta_{h}$ is the halo over-density and $\delta_{m}$ is the matter density perturbation. The EST framework is a very useful tool to calculate the abundance of structures. It is based on the concept of threshold crossing when we go from larger scales to smaller scales with the exclusion of the cloud-in-cloud effect which is present in Press-Schechter formalism[65]. At large scales EST is known to reproduce the initial condition while in small scales it determines the local bias parameter with linear and nonlinear terms [66, 67] which are in reasonably good agreements with numerical simulations [68 70]. It is worth to mention that the halo bias is a function of redshift and scales. This scale-dependance is introduced by applying the initial non-Gaussian condition. According to Appendix $\mathrm{A}$ the large scale halo bias can be treated in peak-background splitting [72]. The idea of splitting of the density contrast to short and long wavelength can be translated to a similar splitting of Bardeen potential (correspondingly the matter density contrast) due to Poisson equation which depends on cosmological parameters. The matter density in PBS can be written as

$$
\rho=\bar{\rho}\left(1+\delta_{s}+\delta_{l}\right) .
$$

The number density of formed structures with mass $m$ can be expressed as a function of small scale statistics, (i.e. smalls scale power spectrum $\left.P_{s}(k)\right)$ and the background long wave-length perturbation $\delta_{l}$ (i.e. $n=n\left[\delta_{l}(\vec{x}), P_{s}\left(k_{s}\right) ; m\right]$ ) 74]. The bias parameter in the context of peak-background splitting (PBS) is described by the fact that the background large scale over-density changes the critical threshold of spherical collapse 71]. Therefor, the criteria for collapse becomes

$$
\delta_{s}>\delta_{c}-\delta_{l},
$$

where $\delta_{s}$ is the matter density contrast of the structure, (the subscript "s" stands for the short wavelength); $\delta_{l}$ is the background (long-wavelength) density contrast and $\delta_{c} \simeq 1.68$ is the critical density contrast in spherical collapse formalism [72, 73] (for a review of PBS see Appendix @ ). Now in order to find the bias parameter we have to compare the dark matter halo abundance, in cases with and without the presence of long wavelength (background) over-density. For this task we use the EST approach. In Appendix A, we review the concept of EST in more details and we will derive the bias parameter using the PBS in EST context.

The primordial potential in Fourier space can be translated into the late time potential as

$$
\Phi(k, z)=\frac{9}{10} \Phi_{i n i} T(k) D(z)(1+z) .
$$

Here $\Phi_{i n i}$ represents the initial Bardeen potential sourced by the inflaton field quantum fluctuations which is related to the curvature perturbation in radiation dominated via $\Phi_{i n i}=2 / 3 \mathcal{R}, T(k)$ is the transfer function and $D(z)$ is the growth function normalized to scale factor at early times. An important point here is that we use the usual formalism of the isotropic linear perturbation theory when we calculate the effects of anisotropic NG on LSS observables. This is reasonable to first order because after inflation ends we recover the isotropic FRW Universe at the background level. The anisotropies are inherited only in seed perturbations which show themselves only through power-spectrum and bispectrum. 
Now one can relate the initial non-Gaussian potential to $\delta_{l}$ via Poisson equation in sub-horizon scale and linearregime

$$
\delta_{l}=M(k, z) \Phi
$$

where

$$
M(k, z)=\frac{3 k^{2} T(k) D(z)}{5 \Omega_{m}^{0} H_{0}^{2}} .
$$

Here, $\Omega_{0}$ and $H_{0}$ are the matter fraction energy density and Hubble parameter at present time, respectively. In order to calculate the halo-bias term, we should calculate the effect of large scale perturbation, $\delta_{l}$, on the Probability Distribution Function (PDF) density fluctuations. This yields the following relation between the Lagrangian halo number density and the PDF of density fluctuations 61]

$$
1+\delta_{h}^{L}=\frac{\partial_{m} \int_{\infty}^{\delta_{c}} \Pi\left(\delta_{s}, \sigma_{m}^{2}, \delta_{c} ; \delta_{l}, \sigma_{l}^{2}\right) d \delta_{s}}{\partial_{m} \int_{\infty}^{\delta_{c}} \Pi_{0}\left(\delta_{s}, \sigma_{m}^{2}, \delta_{c}\right) d \delta_{s}},
$$

where $\Pi\left(\delta_{s}, \sigma_{m}^{2}, \delta_{c} ; \delta_{l}, \sigma_{l}^{2}\right)$ is the conditional PDF of density fluctuations for $\delta_{s}$ with corresponding variance $\sigma_{m}$, when there is a background perturbation of $\delta_{l}$ and variance $\sigma_{l}$. The notation used in the conditional PDF, means that the variance $\sigma_{m}$ at large scales converges to the value $\sigma_{m}=\sigma_{l}$, in contrast to the unconditional PDF of density fluctuations $\Pi_{0}\left(\delta_{s}, \sigma_{m}^{2}, \delta_{c}\right)$ in which the variance vanishes $\left(\sigma_{m} \rightarrow 0\right)$ at large scales. It will be relevant to define a quantity that shows the probability of first up-crossing in the time interval between $\sigma_{m}^{2}$ and $\sigma_{m}^{2}+d \sigma_{m}^{2}$ in EST language as

$$
\mathcal{F}_{0}\left(\delta_{c}, \sigma_{m}^{2}\right) \equiv-\frac{\partial}{\partial \sigma_{m}^{2}} \int_{-\infty}^{\delta_{c}} \Pi_{0}\left(\delta_{s}, \sigma_{m}^{2}, \delta_{c}\right) d \delta_{s}
$$

From the above formalism, we can see the effect of primordial NG on LSS. Assuming that the Bardeen Potential has a local-type NG we have

$$
\Phi=\phi+f_{N L} \phi^{2},
$$

where $\phi$ is the Gaussian field. Now we can use the splitting idea on $\phi$ by applying $\phi=\phi_{l}+\phi_{s}$, where $\phi_{l}$ is the long wavelength mode of potential and $\phi_{s}$ is short wavelength corresponding to the scale of structure. In Appendix $\mathrm{A}$ we discuss how the above non-linear form can be generalized to a model with arbitrary shape of non-Gaussianity, in which case, the non-linear term generalizes into a kernel.

Now in the presence of primordial NG, the modes are not independent and the conditional PDF of density fluctuations is modified by the non-Gaussian long-wavelength mode. In this case the PDF of density fluctuations will be a function of $\phi_{l}$ through the variance and also higher order cumulants $\left(c_{p} \equiv\left\langle\delta_{s}^{p}\right\rangle_{c}\right)$ as

$$
\Pi\left(\delta_{s}, \sigma_{m}^{2}, \delta_{c} ; \delta_{l}, 0\right) \rightarrow \Pi\left[\delta_{s}, \sigma^{2}(\phi), c_{p}(\phi), \delta_{c} ; \delta_{l}(\phi), 0\right] .
$$

The non-Gaussian initial conditions introduces a dependence on higher-order cumulants which does not exist in the Gaussian case. These higher order cumulants depend on the long wavelength mode. Under the assumption that all these effects are small, using the EST formalism we can Taylor expand the conditional PDF of density fluctuations, $\Pi$, around unconditional one, $\Pi_{0}$. The EST formalism with a sharp $k$-space filter and with the assumption of Gaussian initial conditions leads to a Markovian random walk condition for the density contrast value when changing the mass scale/radius in each step. This means that in the case of Markovianity we neglect the environmental dependence in halo formation process. Recently Maggiore and Riotto [75 77] showed how to extend the EST with the pathintegral method to include the non-Markovian condition. Also there are many follow up works where this effect on non-Gaussian halo bias is studied 78 82] (for more details, see appendix @). In this work we study the effects on linear bias from the anisotropic primordial NG and include only the first derivative contribution in Taylor expansion, Eq. A17). As it was shown in Scoccimarro et al. 61], the bias parameter calculated in first order of $f_{N L}$ is not sensitive to the Markovianity/non Markovianity condition. In this work we concentrate on NG at the order of $f_{N L}$. It is worth mentioning that in higher order NG, such as in trispectrum analysis yielding the $g_{N L}$ parameter, non-Markovianity is induced which results in to a new scale-dependence in bias. The analysis in [78 82] show that the departure from Markovian condition in bias parameter is more significant for low mass ranges. As we showed in appendix $\mathrm{A}$ up to 
first order in $f_{N L}$, only the first two terms in Taylor expansion of PDF density fluctuations appear. These terms are derivatives of PDF density fluctuations with respect to the long wavelength mode $\delta_{l}$ and the variance $\sigma_{l}$. The higher order terms, corresponding to derivatives with respect to $c_{p}(p \geq 3)$, contribute to $\mathcal{O}\left(f_{N L}^{2}\right)$ and $\mathcal{O}\left(g_{N L}\right)$ bias.

As mentioned above, in this work we consider only NG at the order of $f_{N L}$. The $p=1$ contribution, the first term in Taylor expansion, Eq. (A18), is the usual scale-independent linear bias from Gaussian perturbations. Keeping in mind $b \equiv \delta_{h} / \delta_{l}$, for the first order linear bias $\left(b_{1 L}\right)$ we have

$$
p=1: \quad b_{1 L}=\frac{\partial_{m} \int\left(\partial \Pi / \partial \delta_{l}\right)_{0}}{\partial_{m} \int \Pi_{0}}=\left[\frac{\partial}{\partial \delta_{l}} \ln \left(\frac{d n\left(\delta_{l}\right)}{d \ln m}\right)\right],
$$

which can be written as:

$$
b_{1 L}=\frac{\partial}{\partial \delta_{l}} \ln \left(n\left(\delta_{l}\right)\right) .
$$

In the presence of primordial non-Gaussianity, there are new contributions from higher order cumulants $p \geq 2$. As a result, the next to leading order term gives

$$
p=2: \quad b_{2 L}=\frac{\partial_{m}\left[I_{21} \int \partial \Pi_{0} / \partial \sigma_{m}^{2}\right]}{M(k) \partial_{m} \int \Pi_{0}},
$$

which in general is a scale-dependent correction to the leading order, scale-independent bias, Eq. A20). The key quantity here is $I_{21}$ which is the derivative of second cumulant $\sigma_{m}^{2},(p=2)$, with respect to the long wavelength mode $\phi_{l}$ which is obtained as 61]

$$
I_{21}(k, m)=\frac{1}{P_{\phi}(k)} \int B_{\hat{\delta} \hat{\delta} \phi}(q, k-q,-k) d^{3} q,
$$

where $B_{\hat{\delta} \hat{\delta} \phi}$ is the cross bispectrum of small-scale smoothed density $\hat{\delta}$ and $\phi$. As a result, $I_{21}$ is the quantity which we are looking for in the case of non-Gaussian initial condition which introduces scale-dependent bias at the order of $\mathcal{O}\left(f_{N L}\right)$.

So far only the Lagrangian bias appeared because peaks are those of the initial density field (linearly extrapolated). Making the standard assumptions that halos move coherently with the underlying dark matter, and using the techniques outlined in [83 86], one can obtain the final Eulerian bias in linear order as

$$
b_{E}=1+b_{1 L}+b_{2 L}
$$

where the linear bias is

$$
b_{1 L}=\frac{2}{\delta_{c}} \partial_{\ln \sigma_{m}^{2}} \ln \left(\sigma_{m}^{2} \mathcal{F}\right)=b_{1 L(G)}+b_{1 L(N G)} .
$$

A very important point is that in above equation we have omitted the subscript of $\mathcal{F}$, which means that the nonGaussianity changes the mass function of the structures so the first linear term will have a contribution from primordial non-Gaussianity. Consequently, we have

$$
b_{1 L(G)}=\frac{2}{\delta_{c}} \partial_{\ln \sigma_{m}^{2}} \ln \left(\sigma_{m}^{2} \mathcal{F}_{0}\right)
$$

and

$$
b_{1 L(N G)}=\frac{2}{\delta_{c}} \partial_{\ln \sigma_{m}^{2}} \ln \left(\sigma_{m}^{2} \mathcal{R}_{N G}\right)=\frac{\partial \ln \mathcal{R}_{N G}\left(m, f_{N L}\right)}{\partial \delta_{l}},
$$

where $\mathcal{F}=\mathcal{R}_{N G} \mathcal{F}_{0}$, and $\mathcal{R}_{N G}$ comes from the deviation of PDF density fluctuations from the Gaussian case [24, 87]. In other words, the effects of non-Gaussianity appeared both in the mass function and in the power spectrum via scale-dependent bias parameter. Since in this work we are interested in the scale-dependence features of bias, the contribution of $\mathcal{R}_{N G}$ is not much of interest. For the Gaussian case we use the Sheth-Tormen [88] Gaussian mass function. For the non-Gaussian mass function effect we use the results of 21] in which the non-Gaussian mass function is expanded in the Press-Schechter framework [20] such that

$$
\mathcal{R}_{N G}\left(m, f_{N L}\right)=1+\frac{1}{6} x\left(x^{2}-3\right) s_{3}(x)-\frac{1}{6}(x-1 / x) \frac{d s_{3}(x)}{d \ln (x)},
$$


where $x \equiv \delta_{c} / \sigma_{M}$ and $\delta_{c}=1.68$ is the critical density and $s_{3}$ is the reduced skewness defined as

$$
s_{3}(R) \equiv \frac{\left\langle\delta_{R}^{3}\right\rangle}{\left\langle\delta_{R}^{2}\right\rangle^{3 / 2}}=\frac{\left\langle\delta_{R}^{3}\right\rangle}{\sigma_{m}^{3}} .
$$

The skewness is related to the matter bispectrum as

$$
\left\langle\delta_{R}^{3}\right\rangle=\int \frac{d^{3} q_{1}}{(2 \pi)^{3}} \frac{d^{3} q_{2}}{(2 \pi)^{3}} W\left(R q_{1}\right) W\left(R q_{2}\right) W\left(R q_{12}\right) M\left(q_{1}, z\right) M\left(q_{2}, z\right) M\left(q_{12}, z\right) B_{0}\left(q_{1}, q_{2}, q_{12}\right),
$$

where $\vec{q}_{12}=-\left(\vec{q}_{1}+\vec{q}_{2}\right), W$ is the window function in Fourier space and $R$ is the smoothing scale. For more details see Appendix A

On the other hand, the scale-dependent bias can be rewritten as

$$
b_{2 L}=\frac{I_{21}(k, m)}{2 \sigma_{m}^{2} M(k, z)} \delta_{c} b_{1 L}+\frac{1}{M(k, z)} \partial_{\ln \sigma_{m}^{2}}\left(\frac{I_{21}(k, m)}{\sigma_{m}^{2}}\right) .
$$

So the total Eulerian bias, up to first order in $f_{N L}$, can be split into the scale-independent term, $b_{s i}$, and scaledependent term, $b_{s d}$, as

$$
b_{t(E)}=b_{s i}+b_{s d}
$$

where $b_{s i}$ and $b_{s d}$ are

$$
b_{s i} \equiv b_{G}+b_{1 L(N G)},
$$

with $b_{G} \equiv 1+b_{1 L(G)}$ and

$$
b_{s d}=b_{2 L}+\mathcal{O}\left(f_{N L}^{2}\right)+\mathcal{O}\left(g_{N L}\right)+\ldots
$$

In next section we find the scale-independent and the scale-dependent bias for general anisotropic initial power spectrum and discuss the effects of the primordial anisotropies on the bias parameter.

\section{GENERAL FORMULATION OF ANISOTROPIC BIAS}

In this section we calculate the bias parameter, assuming a general model independent primordial anisotropy. For this task we write the power spectrum and bispectrum in their most general anisotropic forms. Then we find the possible configurations that give the scale-dependent bias.

As mentioned in section 1 the general anisotropic power spectrum can be written as [60]

$$
P(\vec{k})=P_{0}(k)\left[1+\sum_{L M} g_{L M}(k) Y_{L M}(\hat{k})\right]
$$

which is a generalization of our anisotropic power spectrum defined in Eq.(7), where all the $k$-dependence is buried in the coefficient $g_{L M}$. In this work we assume that the mechanism of spherical collapse for structure formation is still applicable in our model with primordial anisotropic perturbations. This is motivated from the fact that after inflation the background becomes isotropic and anisotropies are encoded only on primordial perturbations. Secondly, the collapse mechanism is a local process so up to first order it is not affected by large scale anisotropies. As a result, we assume that the transfer function at leading order is not affected by the primordial anisotropies. Having this said, it would be interesting to perform the analysis with the above assumptions being relaxed, but this is beyond the scope of this work.

First of all the variance is defined through the linear matter power spectrum

$$
\sigma^{2}(M, z)=\int \frac{d^{3} k}{(2 \pi)^{3}} P_{L}(k) W^{2}(k R),
$$

where $P_{L}$ and $W(k R)$ are the linear matter power spectrum and window function in Fourier space which are discussed in details in the Appendix $\mathrm{A}$. The anisotropic variance reads as

$$
\sigma_{A}^{2}(m, z)=\int d \psi d(\cos \theta) k^{2} d k P_{0}(k, z) W(k R)\left[1+\sum_{L M} g_{L M}(k) Y_{L M}\right] .
$$


The spherical harmonics are orthonormal via

$$
\int Y_{l m} Y_{l^{\prime} m^{\prime}}^{*} d \Omega=\delta_{l l^{\prime}} \delta_{m m^{\prime}}
$$

As a result, noting that $Y_{00}=1 / \sqrt{4 \pi}$, we have

$$
\int Y_{l m} d \Omega=\sqrt{4 \pi} \int Y_{l m} Y_{00}^{*} d \Omega=\sqrt{4 \pi} \delta_{l 0} \delta_{m 0},
$$

so the variance, Eq.(46), can be simplified to

$$
\sigma_{A}^{2}(m, z)=4 \pi \int k^{2} d k P_{0}(k, z) W(k R)\left[1+g_{00}(k) / \sqrt{4 \pi}\right] .
$$

Ignoring the scale-dependence of $g_{00}$, we have

$$
\sigma_{A}^{2}(m, z) \simeq \sigma_{0}^{2}(m, z)\left[1+g_{00} / \sqrt{4 \pi}\right],
$$

with

$$
\sigma_{0}^{2}(m, z)=4 \pi \int k^{2} d k P_{0}(k, z) W(k R) .
$$

Similarly, we can generalize the bispectrum in squeezed limit, where $k_{3} \ll k_{1} \approx k_{2}$, as follows

$$
B_{\zeta}\left(\vec{k}_{1}, \vec{k}_{2}, \vec{k}_{3}\right) \approx 2 \sum_{L, l, m} c_{L l m} \mathcal{P}_{L}\left(\cos \theta_{3}\right) Y_{l, m}\left(\theta_{1}, \psi_{1}\right)\left(P_{0}\left(\vec{k}_{1}\right) P_{0}\left(\vec{k}_{3}\right)\right)
$$

where $\mathcal{P}_{L}$ is the Legendre function, $Y_{l, m}\left(\theta_{1}, \phi_{1}\right)$ are the spherical harmonics, by which the whole direction-dependence of bispectrum is encoded and the $c_{L l m}$ are the $k$-dependent coefficients. This decomposition is possible because we set the $z$ coordinate along the anisotropy direction and rotate the $x-y$ plane in Fourier (k-space) such that the azimuthal angle of $k_{3}$ is set to zero. We can do this rotation because of the symmetry in the $x-y$ plane. This kind of decomposition, i.e. decomposing into a $k$-dependent sector $\left(c_{L l m}\right)$ and the angular-dependent part is especially useful to find the corresponding $I_{21}$ term in the case of generic anisotropic bispectrum and the corresponding scale-dependent bias.

Since in the real space the bispectrum is a real quantity, and noting that the bispectrum above is defined in Fourier space, the following condition holds

$$
c_{L l m}^{*}=(-1)^{L+l} c_{L l-m} .
$$

Now, using Eq.(52), we can write $I_{21}$ as

$$
I_{21}=2 \sum_{L, l, m} \int d \psi_{1} d\left(\cos \theta_{1}\right) q^{2} d q M_{m, z}(q) M_{m, z}(|k-q|) c_{L l m}(q, k) \mathcal{P}_{L}\left(\theta_{3}\right) Y_{l, m}\left(\theta_{1}, \psi_{1}\right) P_{0}(q),
$$

which, again using the orthonormality of spherical harmonics, simplifies to

$$
I_{21}(\mathbf{k}, m, z)=4 \sqrt{\pi} \sum_{L} \mathcal{P}_{L}\left(\cos \theta_{k}\right) \times \int q^{2} d q M_{m, z}(q) M_{m, z}(|k-q|) c_{L, l=0, m=0}(q, k) P_{0}(q) .
$$

This is an interesting result showing that the anisotropic bispectrum induces an anisotropic, scale-dependent bias through the Legendre function of the angle between the anisotropic direction $\hat{n}$ and the long wavelength mode. It is also interesting to note that there is a Selection Rule for scale-dependent bias at $\mathcal{O}\left(f_{N L}\right)$, where the harmonic numbers of the short wavelength must vanish $(l=0, m=0)$. All the other parts of anisotropic bispectrum with $l, m \neq 0$ do not contribute to the leading order scale-dependent bias.

It is worth to mention that the reality assumption of the bispectrum implies

$$
c_{L 00}^{*}=(-1)^{L} c_{L 00},
$$


where in the case of real $c_{L 00}, L$ is even. Now, if we define a direction-independent parameter $I_{(L) 21}^{d i}$ as

$$
I_{(L) 21}^{d i}(k, m, z) \equiv 4 \sqrt{\pi} \times \int q^{2} d q M_{m, z}(q) M_{m, z}(|k-q|) c_{L 00}(q, k) P_{0}(q),
$$

then $I_{21}$ will be re-expressed as

$$
I_{21}\left(k, m, z, \theta_{k}\right)=\sum_{L} \mathcal{P}_{L}\left(\cos \theta_{k}\right) I_{(L) 21}^{d i}(k, m, z)
$$

where $\theta_{k}$ is the angle between $\hat{n}$ and $\vec{k}$. Now the scale-dependent bias becomes

$$
b_{2 L}\left(k, z, m, \theta_{k}\right)=\frac{\sum_{L} \mathcal{P}_{L}\left(\cos \theta_{k}\right) I_{(L) 21}^{d i}(k, m, z)}{2 \sigma_{A}^{2}(m, z) M(k, z)} \delta_{c} b_{1 L}+\frac{1}{M(k, z)} \partial_{\ln \sigma_{A}^{2}}\left(\frac{\sum_{L} \mathcal{P}_{L}(\cos \gamma) I_{(L) 21}^{d i}(k, m, z)}{\sigma_{A}^{2}(m, z)}\right)
$$

where in this case $\sigma_{A}^{2}$ is defined as in Eq. (49).

In next Section, as a specific example of the general formulation presented above, we study our model of anisotropic inflation introduced in Sec. (III), corresponding to $L=2, l=0, m=0$.

\section{ANISOTROPIC BIAS FOR GAUGE FIELD INFLATIONARY MODELS}

In this Section we study the LSS bias in the model of anisotropic inflation as a special example of general formulation developed in previous Section. For a related work with a phenomenological modeling of bispectrum and its implication for bias see [89]. As in the previous Section, we continue with our simplifying assumptions of spherical collapse and take the transfer function to be that of the isotropic background. We investigate the change in PDF of density perturbations and the corresponding cumulants, and the effect of these changes on bias.

In our model the variance is modified due to the fact that we use anisotropic power spectrum. However, we show below that it is not direction-dependent (as it was shown in general case). Without loss of generality, we can assume that the anisotropy is pointed along the $z$-direction in spherical coordinates $(\hat{n}=\hat{z})$. Then, starting from Eq. (7) for the primordial anisotropic power spectrum, we have

$$
\sigma_{A}^{2}(m, z)=\int d \psi d \cos \theta d k k^{2} P_{0}(k, z)\left[1+g_{*}(k) \cos ^{2} \theta\right] W(k R) \simeq\left(1+\frac{g_{*}}{3}\right) \sigma_{m}^{2},
$$

where $\theta$ and $\psi$ are spherical coordinate angles and $g_{*}(k)=-24 I N\left(k_{1}\right) N\left(k_{2}\right)$. Note that $\sigma_{A}^{2}$ is the variance obtained from the full anisotropic power spectrum, where $\sigma_{m}^{2}$ is the variance corresponding to the isotropic part. Since the scale-dependence of $g_{*}$ is logarithmic through $N\left(k_{i}\right)$, as given in Eq. (9), as a first approximation we can ignore its scale-dependence so we have the last approximate equality in Eq. (60). Note that, as we mentioned before, the variance does not have any direction-dependence, which is somewhat an obvious observation, since one should integrate over the full 3D Fourier space to obtain the variance, eliminating any direction present in power spectrum. However, it is interesting to note that depending on the sign of anisotropy parameter $g_{*}$ the correction to the variance due to anisotropy enhances or suppresses the leading order term. From the above variance, we can obtain the leading order scale-independent bias $b_{1 L}$.

As for the next step, we obtain the bias from the anisotropic bispectrum which is both scale-dependent and directiondependent. This would be the main effect of anisotropy in the bias parameter. In order to obtain $b_{2 L}$, we need $I_{21}$ where the information from primordial bispectrum is encoded. We use the squeezed limit $\left(k_{3} \ll k_{2} \simeq k_{1}\right)$ bispectrum predicted by the model, Eq.(13). Because of the symmetry in $x-y$ plane we rotate the long wavelength mode such that $\psi_{\hat{k}_{3}}=0$. Inserting the bispectrum to $I_{21}$, Eq.(32) results in

$$
\begin{aligned}
I_{21}(\mathbf{k}, z, m) & =24 \int d \psi_{q} d\left(\cos \theta_{q}\right) d q q^{2} M_{m}(q, z) M_{m}(|k-q|, z) P_{\phi}(q) N(k)\left|g_{*}(q)\right| \\
& \times\left[1-\cos ^{2} \theta_{q}-\cos ^{2} \theta_{k}+\cos \theta_{q} \cos \theta_{k}\left(\sin \theta_{q} \sin \theta_{k} \cos \psi_{q}+\cos \theta_{q} \cos \theta_{k}\right)\right],
\end{aligned}
$$

where $k$ and $q$ correspond to long and short wavelength ( $k_{3}$ and $k_{2}$ in Eq. (13)), and $\theta$, $\psi$ are polar and azimuthal angles in spherical coordinates, defined by the angle between the anisotropy direction $\hat{n}$ and wavenumbers $\hat{q}$ and $\hat{k}$ respectively

$$
\cos \gamma_{q}=\hat{q} \cdot \hat{n}=\sin \theta_{\hat{n}} \sin \theta_{\hat{q}} \cos \left(\psi_{\hat{n}}-\psi_{\hat{q}}\right)+\cos \theta_{\hat{n}} \cos \theta_{\hat{q}}
$$




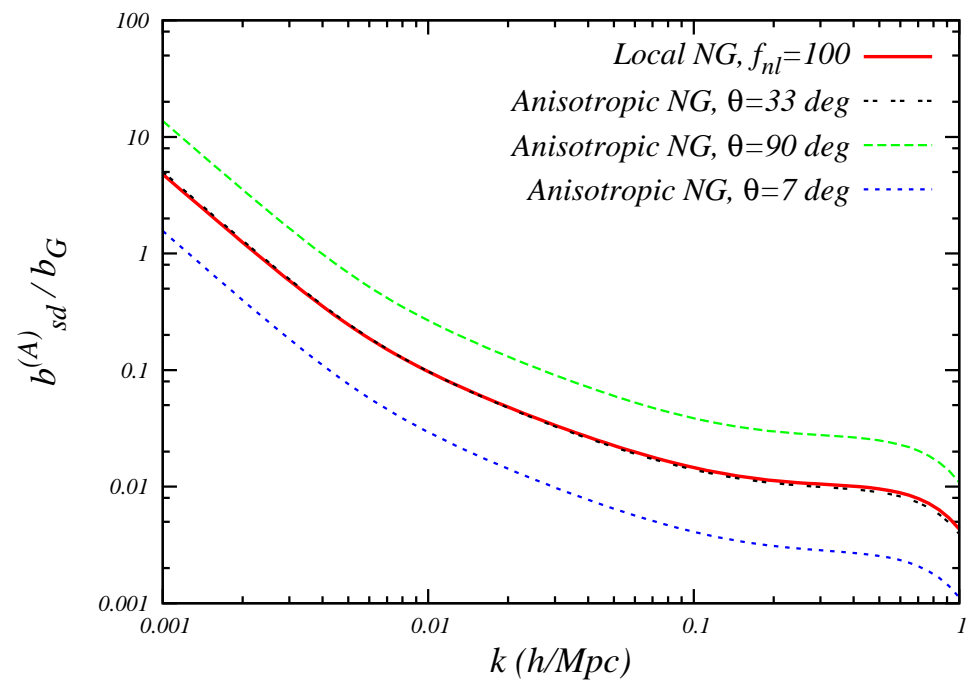

FIG. 1: The fraction of scale-dependent anisotropic bias Eq. (70), to the Gaussian bias, $b_{s d}^{(A)} / b_{G}$, is plotted versus the wavenumber for different angles between the long wavelength mode and the anisotropy direction. The black double-dashed curve, the green long-dashed curve and the blue dotted curve, respectively, are for $\theta_{k}=33^{\circ}, \theta_{k}=90^{\circ}$ and $\theta_{k}=7^{\circ}$. The solid red curve indicates the local non-Gaussianity with $f_{N L}=100$. For the anisotropic bias, we choose $I \simeq 1.9 \times 10^{-6}$ such that $f_{N L}^{e f f}=100$ from Eq. (15). The redshift is $z=0$.

$$
\cos \gamma_{k}=\hat{k} \cdot \hat{n}=\sin \theta_{\hat{n}} \sin \theta_{\hat{k}} \cos \left(\psi_{\hat{n}}-\psi_{\hat{k}}\right)+\cos \theta_{\hat{n}} \cos \theta_{\hat{k}}
$$

Now we can integrate the angular dependence $\gamma_{q}$ which yields

$$
I_{21}\left(k, z, m, \theta_{k}\right)=64 \pi N(k)\left(1-\cos ^{2} \theta_{k}\right) \int d q q^{2} M_{m, z}(q) M_{m, z}(|k-q|) P_{\phi}(q)\left|g_{*}(q)\right| .
$$

It is interesting to note that the orientation-dependence appears in the form of $\sin ^{2} \theta_{k}$. As a result, the bias vanishes when $\sin \theta_{k}=0$, i.e. when the long wavelength mode is aligned with the anisotropic direction. This result originates from the fact that in this specific direction the bispectrum vanishes in squeezed limit as one can check from Eq. (13). Furthermore, since in the model under consideration $g_{*}$ has a mild scale-dependence via logarithmic correction in $N(k)$, we observe that there is an extra but mild k-dependent factor in $I_{21}$ in comparison with the standard local non-Gaussian shape [61, 90]. Besides that, $I_{21}$ linearly depends on $I$ which is the free parameter of the anisotropic inflationary model. Now, by using the variance and $I_{21}$ parameter obtained above we can find the bias parameter in the anisotropic model by

$$
b_{1 L}=\frac{\partial_{m} \int\left(\partial \Pi / \partial \delta_{l}\right)_{0}}{\partial_{m} \int \Pi_{0}}=\left[\frac{\partial}{\partial \delta_{l}} \ln \left(\frac{d n\left(\delta_{l}\right)}{d \ln m}\right)\right]=b_{1 L(G)}+b_{1 L(N G)},
$$

where, for the first order linear bias in the case of anisotropy, we have

$$
b_{(A) 1 L(N G)}=\frac{2}{\delta_{c}} \partial_{\ln \sigma_{A}^{2}} \ln \left(\sigma_{A}^{2} \mathcal{R}_{(A) N G}\right)=\frac{\partial \ln \mathcal{R}_{(A) N G}\left(m, f_{N L}\right)}{\partial \delta_{l}},
$$

in which the subscript $(A)$ has been added to point out that the parameters are obtained in the presence of anisotropy. Here $\mathcal{R}_{(A) N G}$ is the anisotropic non-Gaussian correction to the PDF of density fluctuations defined by

$$
\mathcal{R}_{(A) N G}\left(m, f_{N L}\right)=1+\frac{1}{6} x_{A}\left(x_{A}^{2}-3\right) s_{(A) 3}\left(x_{A}\right)-\frac{1}{6}\left(x_{A}-1 / x_{A}\right) \frac{d s_{3}\left(x_{A}\right)}{d \ln \left(x_{A}\right)},
$$

where $x_{A} \equiv \delta_{c} / \sigma_{A}$ and $\delta_{c}=1.68$ is the critical density and $s_{(A) 3}$ is the reduced skewness defined as

$$
s_{(A) 3}(R) \equiv \frac{\left\langle\delta_{R}^{3}\right\rangle}{\left\langle\delta_{(A) R}^{2}\right\rangle^{3 / 2}}=\frac{\left\langle\delta_{(A) R}^{3}\right\rangle}{\sigma_{A}^{3}} .
$$




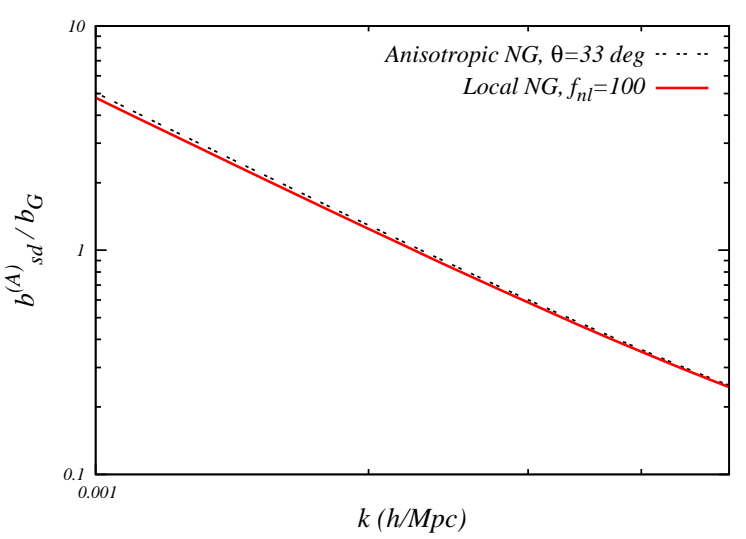

FIG. 2: This figure is a zoom-in of Fig. 1 for long wavelength modes where we compare $b_{s d}^{(A)} / b_{G}$ in our model with $\theta_{k}=33^{\circ}$ to the local non-Gaussian model with $f_{N L}=100$. This shows small deviation between the two models due to a mild scaledependence of $g_{*}$ parameter.

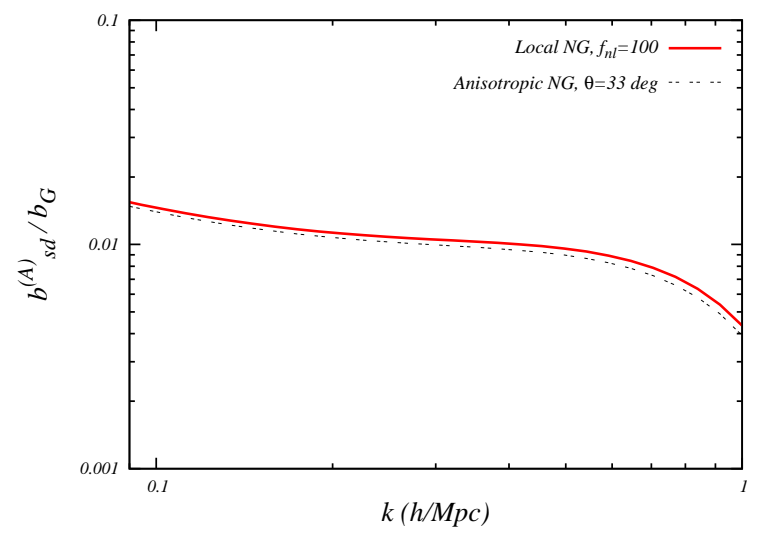

FIG. 3: This figure is a zoom-in of Fig. 1 for short wavelength modes where again we compare $b_{s d}^{(A)} / b_{G}$ in our model for $\theta_{k}=$ $33^{\circ}$ to the local non-Gaussian model with $f_{N L}=100$.

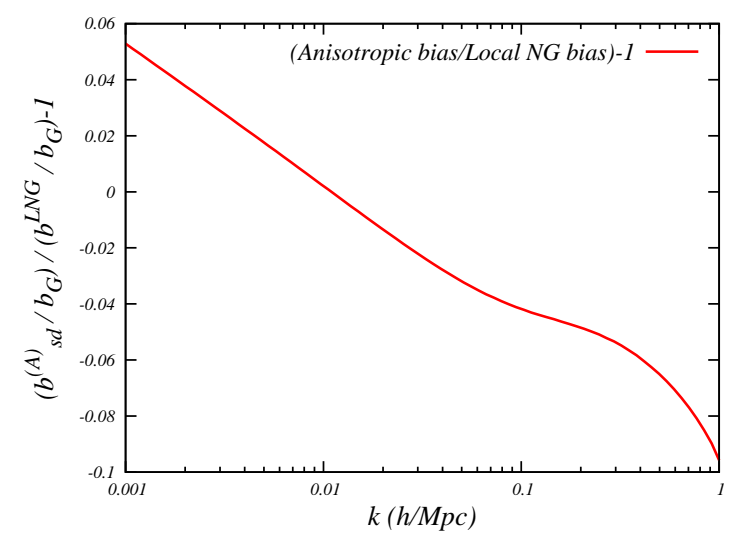

FIG. 4: This figure shows the relative magnitude of the scaledependent bias parameter in our anisotropic model with $\theta_{k}=$ $33^{\circ}$ compared to the local non-Gaussian model with $f_{N L}=100$

Note that, in the above formula, we have to use the full variance $\sigma_{A}$ including the correction due to anisotropy. The anisotropic skewness is also defined as

$$
\left\langle\delta_{(A) R}^{3}\right\rangle=\int \frac{d^{3} q_{1}}{(2 \pi)^{3}} \frac{d^{3} q_{2}}{(2 \pi)^{3}} W\left(R q_{1}\right) W\left(R q_{2}\right) W\left(R q_{12}\right) M\left(q_{1}, z\right) M\left(q_{2}, z\right) M\left(q_{12}, z\right) B_{A}\left(q_{1}, q_{2}, q_{12}\right),
$$

where $B_{A}$ is the anisotropic bispectrum.

The next order term in bias will give the scale-dependent and direction-dependent effect

$$
b_{s d}^{(A)}=b_{(A) 2 L}\left(k, z, m, \theta_{k}\right)=\frac{I_{21}(k, z, m, \theta)}{2 \sigma_{A}(m, z)^{2} M(k, z)} \delta_{c} b_{1 L}+\frac{1}{M(k, z)} \partial_{\ln \sigma_{A}^{2}}\left(\frac{I_{21}\left(k, m, \theta_{k}\right)}{\sigma_{A}^{2}(m, z)}\right) .
$$

So the total Eulerian anisotropic bias is defined as

$$
b_{t(E)}=1+b_{1 L(G)}+b_{(A) 1 L(N G)}+b_{(A) 2 L} .
$$

The first three terms above gives the scale-independent bias, whereas the last term is scale-dependent and directiondependent bias due to the anisotropic bispectrum. Since the model we consider has a bispectrum shape very close to the standard local non-Gaussian shape and since $b_{1 L(N G)}$ is small in comparison with $b_{s d}$ in local shape, 90], we can ignore $b_{1 L(N G)}$ term in our analysis.

In Fig. 1 we plot the relative magnitude of the scale-dependent bias Eq. (70) to Gaussian bias, $b_{(s d)}^{(A)} / b_{G}$, versus the wavenumber. As expected we have approximately $k^{-2}$ scale-dependence similar to the local non-Gaussian shape. 


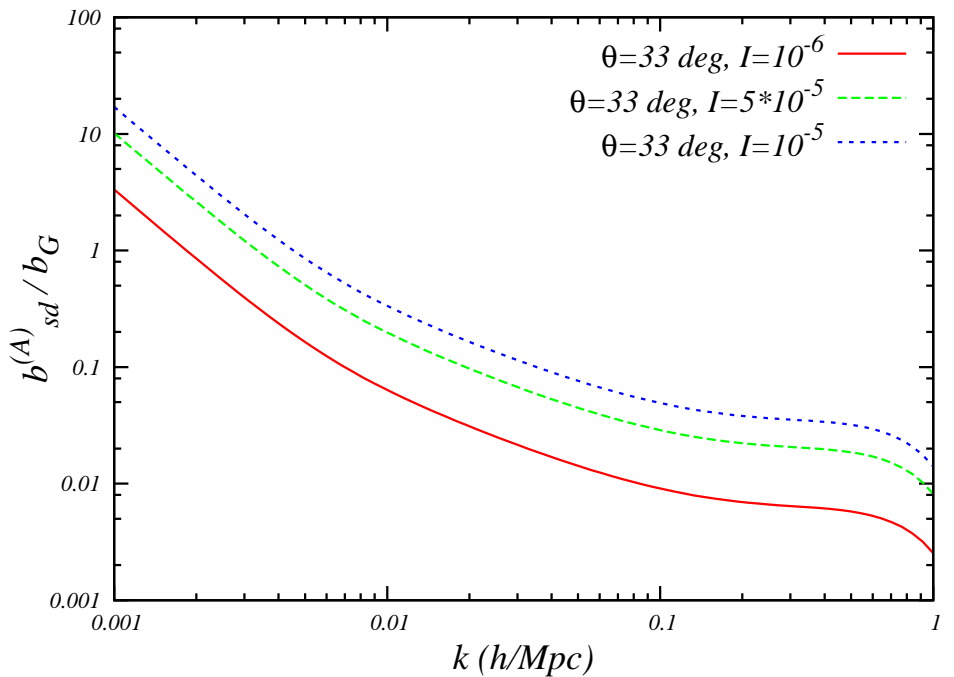

FIG. 5: $b_{s d}^{(A)} / b_{G}$ versus wavenumber is plotted with $\theta_{k}=33^{\circ}$ for three different values of $I$. The solid red curve, the green dashed curve and blue dotted curve, respectively, are for $I=10^{-6}, I=5 \times 10^{-6}$ and $I=10^{-5}$. In all cases we set the redshift to $\mathrm{z}=0$.

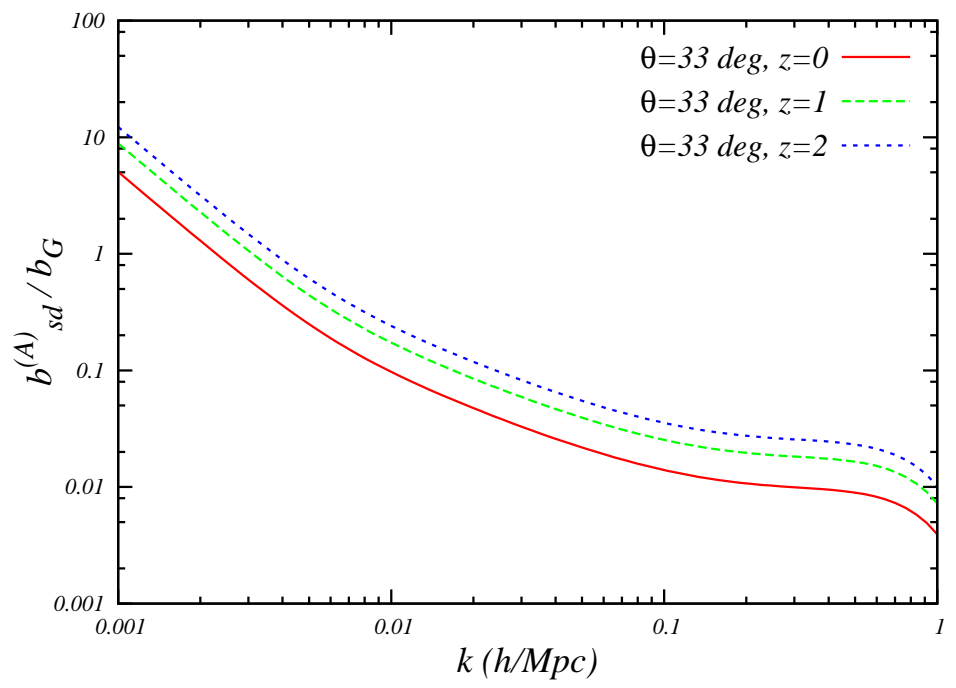

FIG. 6: $b_{s d}^{(A)} / b_{G}$ versus wavenumber is plotted with $I=10^{-6}$ and $\theta_{k}=33^{\circ}$ for three different redshifts. The solid red curve, the green dashed curve and the dotted blue curve, respectively, are for $z=0, z=1$ and $z=2$.

However, this $k$-dependence is slightly different because of mild dependence of $g_{*}$ to wavenumber. A completely new feature is the direction-dependence of bias originated form primordial anisotropy, proportional to $\sin ^{2} \theta_{k_{3}}$. In the case of $\theta_{k_{3}}=\pi / 2$ we have the maximum scale-dependent bias, whereas at angles $\theta_{k_{3}}=0, \pi$, as the anisotropic bispectrum vanishes, the scale-dependent bias also vanishes accordingly.

In order to compare our results with the conventional local non-Gaussian models, we set $\theta_{k_{3}}=33^{\circ}$ to have $f_{N L}^{e f f} \simeq 100$ and then compare the bias in this specific direction with the bias in local shape with amplitude $f_{N L}=100$. In Fig 2 and Fig. 3, for both long and short wavelengths, we compare the fraction of scale-dependent bias, $b_{(s d)} / b_{G}$, from local non-Gaussianity compared to our anisotropic model. In Fig. 4 we compare their relative magnitudes. Since $g_{*}^{1 / 2} \propto N(k)=N_{C M B}+\ln \left(k / k_{C M B}\right)$ the bias of our model is slightly higher than local non-Gaussian case for long wavelength modes while it is slightly lower for short wavelength modes.

The free parameter of our model is $I$. In Fig. 回 we plot $b_{(s d)}^{(A)} / b_{G}$ versus wave number for different values of $I$. The $I$-dependence of bias is linear according to Eq. (64). In Fig. 6 we plot the redshift-dependence of $b_{(s d)}^{(A)} / b_{G}$. As can be seen, at higher redshifts we have higher bias. This is the standard expectation, since at earlier times the non-linearity 
of local, small scale perturbations was weaker and thus they are more sensitive to large scale perturbations, resulting in larger bias.

\section{CONCLUSION AND DISCUSSIONS}

The large scale structure observations, like the statistics of rare objects and the scale-dependence of bias parameter, can be used as complementary cosmological observations to CMB data to constrain the inflationary models. In this work we obtained the scale-dependent and the direction-dependent bias of dark matter halos in anisotropic inflationary models.

The anisotropic model studied in this work has a bispectrum shape very close to the local non-Gaussian shape with an extra mild $k$-dependence and also a direction-dependence. We showed that the bias parameter is mainly influenced by the angle $\left(\theta_{k_{3}}\right)$ between the anisotropy direction and the long wavelength mode $\vec{k}_{3}$ in the squeezed limit by a factor of $\sin ^{2} \theta_{k_{3}}$. An interesting observation is that the scale-dependent bias vanishes at linear order along the direction $\theta_{k_{3}}=0, \pi$. This can be explained by the fact that the bispectrum vanishes in squeezed limit when the long wavelength mode is aligned with the anisotropy direction. This means that, at the level of bispectrum, the model reduces to the Gaussian model along this direction.

As it is clear from the PBS formalism, the bias parameter is mainly affected by the long wavelength mode. We can see this explicitly in our work where the angle between the anisotropy direction and the long mode appears in the bias formula.

The free parameter of our model is $I$. Constraints from the CMB and LSS observations require $I \lesssim 10^{-5}[55[58]$. Roughly speaking, our bias parameter analysis also imply the same order of magnitude for $I$. Considering the fact that the observations are now in good agreement with a Gaussian bias we expect that in quasi-linear regime (where we have the strict constraint on bias) the ratio $b_{s d}^{(A)} / b_{G}$ is at the order of one so from our results we also find $I \lesssim 10^{-5}$.

In this work we also formulated the general, model-independent anisotropic scale-dependent bias at linear order. For this purpose, by assuming the rotational symmetry in $x-y$ plane of Fourier space, we modeled the bispectrum as a function of spherical harmonics, $Y_{l m}$, and the Legendre functions, $\mathcal{P}_{L}$, of the angles between the short/long wavelength modes and the anisotropy direction. Interestingly, we find a selection rule for scale-dependent anisotropic bias, which shows that the bias parameter only responses to the $Y_{00}$ part of the anisotropic bispectrum. We also show that $b_{s d}$ is independent of short wavelength mode and its direction. This is an obvious check for the formalism, since the bias should be only a function of the long wavelength mode.

In this work we assumed that the mechanism of spherical collapse and the conventional form of the transfer function are still applicable. This is motivated from the fact that the collapse of structures is a local mechanism which is less affected by the large scale anisotropies. Having this said in principle it is an interesting question to see how one can generalize the spherical collapse mechanism in the presence of primordial anisotropic fluctuations. On the other hand, the modification of the transfer function due to Boltzmann and perturbed Einstein equations in the presence of an anisotropic cosmic fluid is an interesting question which is beyond the scope of this work.

A very interesting question to ask is whether the ideas presented in this work can be used observationally to find the fingerprints of NG and anisotropies in LSS data. The future LSS surveys such as the Large Synoptic Survey Telescope (LSST) is designed to obtain photometric redshift for almost 4 billion galaxies. The galaxies are distributed in redshift space with the distribution peaking around $\mathrm{z}=1$. This survey enables us to determine the galaxy bias with high accuracy. The galaxy cluster count with combination of other cosmological observations, such as the weak gravitational lensing and CMB data, can measure the full sky bias parameter in the redshift range between 0 to 1 , with a precision as good as $2 \%$ accuracy [97]. However, the challenge from observational side is that if we want to determine the amplitude of the direction-dependent bias we need almost a full sky survey with enough statistics in each patch. This is the most important obstacle in determining the bias via galaxy cluster counting. There are other promising observations, such as the $21 \mathrm{~cm}$ hydrogen intensity power-spectrum, which can be used to detect the fingerprints of NG and anisotropy. It is easier to have a full map data on the hydrogen intensity map although the astrophysical uncertainties will interfere in determining the bias parameter 98 101]. The statistical analysis and future observational forecasts are potential extensions of this work to quantify the chance of the detection. The other point to mention is that the strength of the anisotropy signal depends on $f_{N L}$, the anisotropy parameter $I$ and the angle $\theta_{k}$. Now we have a strong constrain on the local NG from PLANCK data [14] $f_{N L}=2.7 \pm 5.8$ making the signal small. However there are two crucial points here. First, there is a degeneracy between the parameters $f_{N L}, I$ and $\theta_{k}$ and second, the local NG measured by PLANCK collaboration is on large scales (CMB scale) and there is a room for running of $f_{N L}$ towards the smaller scales (sub CMB/LSS scales) making $f_{N L}$ large enough for our analysis. 


\section{Acknowledgments}

We would like to thank Razieh Emami for many insightful discussions on anisotropic inflation. We also thank the anonymous referee for the careful and insightful comments on the draft which were very helpful to improve the presentations.

\section{Appendix A: Halos bias in the Peak Background Splitting in the context of Excursion Set Theory}

In this appendix first we review the Excursion Set theory (EST) then we apply the Peak Background Splitting (PBS) in EST context and finally we argue that how we can find the halo bias. In the context of EST halo formation can be described as the random walk of matter density contrast as the smoothing radius goes from very large radius, corresponding to infinitesimal variance $\sigma^{2}$ and small $\delta$, to the scales crossing the linear threshold for collapse $\delta_{c}$ at some finite smoothing radius. This radius is related to the scale in which the halos form. Within the EST formulation the number density of collapsed objects (dark matter halos of mass $m$ ) per unit mass is given by

$$
\left(\frac{d n}{d m}\right)=\frac{\bar{\rho}}{m} \partial_{m} \int_{-\infty}^{\delta_{c}} \Pi_{0}\left(\delta_{s}, \sigma_{m}^{2}, \delta_{c}\right) d \delta_{s},
$$

where $\sigma_{m}^{2}$ is the variance of the small scale density field smoothed with filter (window function) at spatial scale $R$ and $\bar{\rho}$ is the background energy density, relating the halo mass to smoothing radius by $m=4 \pi \bar{\rho} R^{3} / 3$. Furthermore, $\Pi_{0}\left(\delta_{s}, \sigma_{m}^{2}, \delta_{c}\right)$ is the unconditional probability distribution function (PDF of density fluctuations) of small scale perturbations reaching $\delta_{s}$ (short wavelength density contrast) at variance $\sigma^{2}$. By the subscript 0 as well as the unconditional assumption for PDF of density fluctuations, we mean that the initial condition (first step in random walk) is $\delta_{s}=0$ where $\sigma_{m}^{2}=0$, and it satisfies the absorbing barrier condition $\Pi_{0}\left(\delta_{c}, \sigma_{m}^{2}, \delta_{c}\right)=0$.

The smoothing procedure will be done by top-hat window function in Fourier space [91]

$$
W(x)=\frac{3(\sin x-x \cos x)}{x^{3}},
$$

where $x=k R, k$ is the wavenumber and $R$ is Lagrangian radius of collapsed objects, related to the mass via

$$
R=\left[\frac{m}{1.162 \times 10^{12} h^{2} M_{\odot} \Omega_{m}^{0}}\right]^{1 / 3} M p c
$$

in which $m$ is the mass of the structure and $\Omega_{m}=\Omega_{m}^{0}(1+z)^{3}$.

Since the variance $\sigma_{m}$ is a monotonic function of mass scale due to matter power spectrum we use $\sigma(M)$ as the 1-D variable in random walk. So it will be relevant to define a quantity that shows the probability of first up-crossing in the time $\sigma_{m}^{2}$ and $\sigma_{m}^{2}+d \sigma_{m}^{2}$ as

$$
\mathcal{F}_{0}\left(\delta_{c}, \sigma_{m}^{2}\right) \equiv-\frac{\partial}{\partial \sigma_{m}^{2}} \int_{-\infty}^{\delta_{c}} \Pi_{0}\left(\delta_{s}, \sigma_{m}^{2}, \delta_{c}\right) d \delta_{s} .
$$

Now the number density of collapsed objects will be

$$
\left(\frac{d n}{d m}\right)=\frac{\bar{\rho}}{m}\left|\frac{d \sigma_{m}^{2}}{d m}\right| \times \mathcal{F}_{0}\left(\delta_{c}, \sigma_{m}^{2}\right) .
$$

It is worth to mention that the number density of dark matter halos obeys the normalization condition

$$
\int\left(\frac{d n}{d m}\right) m d m=\bar{\rho}
$$

The key parameter here is the matter density variance which is related to the linear matter power spectrum as

$$
\sigma^{2}(M, z)=\int \frac{d^{3} k}{(2 \pi)^{3}} P_{L}(k) W^{2}(k R),
$$

where the density contrast power spectrum is 91 .

$$
P_{L}(k)=A k^{n_{s}} T^{2}(k) D^{2}(z) .
$$


Here $n_{s}$ is the spectral index, $A$ is the linear matter primordial power spectrum amplitude in $k=0.002 h^{-1} M p c, T(k)$ is the transfer function and $D(z)$ is the growth factor normalized to scale factor at early times.

The evolution of density contrast is imprinted in growth function $D(z)$ and the transfer function $T(k)$, showing the scale-dependence of gravitational potential during cosmic evolution. In this work we use the growth function of standard $\Lambda C D M$ cosmology and the transfer function of Bardeen, Bond, Kaiser and Szalay (BBKS) [73] respectively as below

$$
D(z)=\frac{5}{2} \Omega_{m}\left[\Omega_{m}^{4 / 7}-\Omega_{\Lambda}+\left(1+\frac{\Omega_{m}}{2}\right)\left(1+\frac{\Omega_{\Lambda}}{70}\right)\right]^{-1},
$$

and

$$
T\left(k=q \Omega_{m}^{0} h^{2} M p c^{-1}\right) \approx \frac{\ln [1+2.34 q]}{2.34 q} \times\left[1+3.89 q+(16.2 q)^{2}+(5.47 q)^{3}+(6.71 q)^{4}\right]^{-1 / 4} .
$$

where $\Omega_{m}=\Omega_{m}^{0} a^{-3} /\left(\Omega_{m}^{0} a^{-3}+\Omega_{\Lambda}\right)$ and $\Omega_{\Lambda}=\Omega_{\Lambda}^{0} /\left(\Omega_{m}^{0} a^{-3}+\Omega_{\Lambda}\right)$. We define the growth function, in a way that is normalized to scale factor in deep matter dominated era. An important point to note is that the variance is linearly dependent to growth function due to Eq. (A8) where $\sigma^{2}(M, z)=\sigma^{2}(M, z=0)[D(z) / D(z=0)]^{2}$, where $\sigma^{2}(M, z=0)$ is the present value of variance. This redshift-dependance is important in the sense that the statistics of structure and even the bias parameter will be redshift-dependent. In Fig. (6) we showed the redshift-dependence of bias parameter for our specific anisotropic inflation model. In the matter dominated era $D(z)$ scales like $1 /(1+z)$ which is a decreasing function with respect to redshift. Now that we set the matter density variance and large scale statistics of matter distribution, by setting the initial condition of perturbations we can find the mass function of structures in the Universe. For the Gaussian initial condition the probability distribution function (PDF of density fluctuations) in Fourier-space top-hat filter is [64]

$$
\Pi_{0}\left(\delta_{s}, \sigma_{m}^{2}, \delta_{c}\right)=\mathcal{P}_{G}\left(\delta_{s}, \sigma_{m}^{2}\right)-\mathcal{P}_{G}\left(2 \delta_{c}-\delta_{s}, \sigma_{m}^{2}\right),
$$

where $\mathcal{P}_{G}$ is the Gaussian PDF of density fluctuations.

The assumption of the universality of mass-function (like Press-Schechter) yields

$$
\Pi_{0}\left(\delta_{s}, \sigma_{m}^{2}, \delta_{c}\right)=F\left(\frac{\delta_{s}}{\sigma_{m}}, \frac{\delta_{c}}{\sigma_{m}}\right),
$$

which leads us to write $\sigma_{m}^{2} \mathcal{F}_{0}$ just as a function of threshold quantity $\nu=\delta_{c} / \sigma_{m}$

$$
\sigma_{m}^{2} \mathcal{F}_{0}\left(\delta_{c}, \sigma_{m}^{2}\right)=\frac{\nu f(\nu)}{2}
$$

where $f(\nu)$ is the usual Gaussian factor in Press-Schechter theory [20]. Now in order to find the halo bias in Gaussian and non-Gaussian inflationary models, first we discuss the PBS. We will show how this method is applicable for non-Gaussian fields.

For inflationary models with primordial non-Gaussianity, the gravitational potential is usually written as [92? ,93]

$$
\Phi(x)=\phi(x)+f_{N L}\left([\phi(x)]^{2}-\left\langle\phi^{2}\right\rangle\right)
$$

where $\phi$ is a Gaussian random field. The above relation is the simplest extension of Bardeen potential to include nonlinearity, called local non-Gaussianity. We can generalize the local type non-Gaussianity to arbitrary shape, simply by replacing the non-linear term by a kernel [61]

$$
\Phi(x)=\phi(x)+f_{N L} K[\phi(x), \phi(x)] .
$$

In order to obtain the bias parameter, we can use the PBS idea on the Gaussian potential $\phi=\phi_{s}+\phi_{l}$, where $\phi_{l}$ is the long-wavelength mode of the potential and $\phi_{s}$ is the short-wavelength mode. Since the large scale perturbations change the background for small scale modes, the PDF of density fluctuations for small scale perturbations is modified to the conditional PDF of density fluctuations, $\Pi\left(\delta_{s}, \sigma_{m}^{2}, \delta_{c} ; \delta_{l}, \sigma_{l}^{2}\right)$. In conditional PDF of density fluctuations, the initial condition is $\sigma_{m} \rightarrow \sigma_{l}$ in large scale limit (first step in random walk), in contrast with the unconditional case in which the variance vanishes on large scales.

One of the important assumptions in PBS method is the Markovianity where we can write

$$
\Pi\left(\delta_{s}, \sigma_{m}^{2}, \delta_{c} ; \delta_{l}, 0\right) \approx \Pi\left(\delta_{s}-\delta_{l}, \sigma_{m}^{2}, \delta_{c}-\delta_{l}\right)
$$


where $P i$ is the PDF of density perturbations. The Markovianity condition let us to change the threshold of critical density and density fluctuation of the structure by $\delta_{l}$. In this work we are in he regime where we can neglect the non-Markovianity induced from the primordial NG (for an extended discussion refer to Scoccimarro et al. 61]).

On the other hand, the existence of any type of NG modifies the PDF of density fluctuations in a way that it is no longer independent of higher cumulants, while in Gaussian case the PDF of density fluctuations is completely determined by zeroth order $\left(\delta_{s}\right)$ and first order $\left(\sigma_{m}^{2}\right)$ cumulants. By the splitting procedure explained above, and knowing that the PDF of density fluctuations is now a function of all cumulants, we can expand the Lagrangian halo over-density as an expansion over large-scale $\phi_{l}$ modes [61],

$$
\delta_{h}^{L}=\int d^{3} k \frac{\partial_{m} \int_{-\infty}^{\delta_{c}} d \delta_{s}\left(D \Pi / D \phi_{l}\right)_{0}}{\partial_{m} \int_{-\infty}^{\delta_{c}} d \delta_{s} \Pi_{0}\left(\delta_{s}, \sigma^{2}, \delta_{c}\right)} \phi_{l}+\frac{1}{2} \iint d^{3} k_{1} d^{3} k_{2} \frac{\partial_{m} \int_{-\infty}^{\delta_{c}} d \delta_{s}\left(D^{2} \Pi / D \phi_{l} D \phi_{l}\right)_{0}}{\partial_{m} \int_{-\infty}^{\delta_{c}} d \delta_{s} \Pi_{0}\left(\delta_{s}, \sigma^{2}, \delta_{c}\right)} \times \phi_{l}\left(\vec{k}_{1}\right) \phi_{l}\left(\vec{k}_{2}\right)+\ldots
$$

where $(\ldots)_{0}$ means that the corresponding quantity is evaluated at $\phi_{l}=0$. Note that because the background is now modified by large scale perturbation $\phi_{l}$, the cumulants are now functions of $\phi_{l}$. The first derivative in Eq. A17), to all orders in primordial NG, is

$$
\left(\frac{D \Pi}{D \phi_{l}(\vec{k})}\right)_{0}=\sum_{p=1}^{\infty}\left(\frac{\partial \Pi}{\partial c^{(p)}}\right)_{0}\left(\frac{D c^{(p)}}{D \phi_{l}(k)}\right)_{0}=\left(\frac{\partial \Pi}{\partial \delta_{l}}\right)_{0}\left(\frac{D \delta_{l}}{D \phi_{l}(\vec{k})}\right)+\sum_{p=2}^{\infty} \frac{\partial \Pi_{0}}{\partial c_{m}^{(p)}}\left(\frac{D c^{(p)}}{D \phi_{l}(\vec{k})}\right)_{0},
$$

where the cumulants are defined by

$$
c^{(1)} \equiv \delta_{l}, \quad c_{m}^{(2)} \equiv \sigma_{m}^{2}, \quad c^{(2)} \equiv \sigma^{2}\left(\phi_{l}\right), \quad c_{m}^{(p)} \equiv\left\langle\delta_{s}^{p}\right\rangle_{c}, \quad c^{(p)} \equiv\left\langle\delta_{s}^{p}\left(\phi_{l}\right)\right\rangle_{c}
$$

in which the subscript $m$ for cumulants show that they are evaluated in the absence of the background $\phi_{l}$ (independent of $\left.\phi_{l}\right)$. It is worth to indicate that to first order in $f_{N L}$, only the first two terms in the Taylor expansion above $(p=1,2)$ contributes to the bias, while $p=3$ contributes to $\mathcal{O}\left(f_{N L}^{2}\right)$ and $\mathcal{O}\left(g_{N L}\right)$.

Now we can obtain the bias parameter, using the above formulation. The $p=1$ contribution is the usual scaleindependent bias presented in initially Gaussian case. Keeping in mind $b \equiv \delta_{h} / \delta_{l}$, we have

$$
p=1: \quad b_{1 L}=\frac{\partial_{m} \int\left(\partial \Pi / \partial \delta_{l}\right)_{0}}{\partial_{m} \int \Pi_{0}}=\left[\frac{\partial}{\partial \delta_{l}} \ln \left(\frac{d n\left(\delta_{l}\right)}{d \ln m}\right)\right],
$$

which can be simplifies as

$$
b_{1 L}=\frac{\partial}{\partial \delta_{l}} \ln \left(n\left(\delta_{l}\right)\right) .
$$

The $p=2$ contribution is the scale-dependent correction to the leading order bias coming from primordial NG

$$
p=2: \quad b_{2 L}=\frac{\partial_{m}\left[I_{21} \int \partial \Pi_{0} / \partial \sigma_{m}^{2}\right]}{M(k) \partial_{m} \int \Pi_{0}} .
$$

The quantity $I_{21}$ includes the information about the primordial NG which is the derivative of second cumulant $\sigma_{m}^{2}$, $(\mathrm{p}=2)$, with respect to long wavelength mode $\phi_{l}$ which is obtained to be [61]:

$$
I_{21}(k, m)=\frac{1}{P_{\phi}(k)} \int B_{\hat{\delta} \hat{\delta} \phi}(q, k-q,-k) d^{3} q,
$$

where $B_{\hat{\delta} \hat{\delta} \phi}$ is the cross bispectrum of small-scale smoothed density $\hat{\delta}$ and $\phi$.

So far only the Lagrangian bias appeared in our analysis because the peaks are those of the initial density field (linearly extrapolated). By the standard assumptions that halos move coherently with the underlying dark matter, and using the techniques outlined in [83 86], one can obtain the final Eulerian bias as

$$
b_{E}=1+b_{1 L}+b_{2 L},
$$

Note that, due to the existence of primordial NG, the leading order scale-independent bias also modifies as

$$
b_{1 L}=\frac{2}{\delta_{c}} \partial_{\ln \sigma_{m}^{2}} \ln \left(\sigma_{m}^{2} \mathcal{F}\right)=b_{1 L(G)}+b_{1 L(N G)},
$$


where in Eq. A25 we have omitted the subscript of $\mathcal{F}$, which means that the NG will change the mass function, resulting in a modification of $b_{1 L}$. As a result we have

$$
b_{1 L(G)}=\frac{2}{\delta_{c}} \partial_{\ln \sigma_{m}^{2}} \ln \left(\sigma_{m}^{2} \mathcal{F}_{0}\right)
$$

and

$$
b_{1 L(N G)}=\frac{2}{\delta_{c}} \partial_{\ln \sigma_{m}^{2}} \ln \left(\sigma_{m}^{2} \mathcal{R}_{N G}\right)=\frac{\partial \ln \mathcal{R}_{N G}\left(m, f_{N L}\right)}{\partial \delta_{l}},
$$

as described in Section (III).

$$
\mathcal{R}_{N G}\left(m, f_{N L}\right)=1+\frac{1}{6} x\left(x^{2}-3\right) s_{3}(x)-\frac{1}{6}(x-1 / x) \frac{d s_{3}(x)}{d \ln (x)},
$$

where $x \equiv \delta_{c} / \sigma_{M}, \delta_{c}=1.68$ is the critical density and $s_{3}$ is the reduced skewness defined as

$$
s_{3}(R) \equiv \frac{\left\langle\delta_{R}^{3}\right\rangle}{\left\langle\delta_{R}^{2}\right\rangle^{3 / 2}}=\frac{\left\langle\delta_{R}^{3}\right\rangle}{\sigma_{m}^{3}} .
$$

The skewness is related to the matter bispectrum as

$$
\left\langle\delta_{R}^{3}\right\rangle=\int \frac{d^{3} q_{1}}{(2 \pi)^{3}} \frac{d^{3} q_{2}}{(2 \pi)^{3}} W\left(R q_{1}\right) W\left(R q_{2}\right) W\left(R q_{12}\right) M\left(q_{1}, z\right) M\left(q_{2}, z\right) M\left(q_{12}, z\right) B_{0}\left(q_{1}, q_{2}, q_{12}\right),
$$

where $\vec{q}_{12}=-\left(\vec{q}_{1}+\vec{q}_{2}\right)$ and $W(k R)$ is the window function in Fourier space, smoothing perturbations up to scale $R$. In obtaining the mass function of non-Gaussianity model in this approximation, we have assumed that all the deviation is imprinted in the skewness which may not be entirely true. In order to improve the results, numerical simulations are done [25, 75, 94]. Consequently, a scaling parameter $\kappa$ defined by $R_{N G}(x) \rightarrow R_{N G}(\kappa x)$ are introduced where, in the work of [33], from simulation of [95], it is obtained to be $\kappa=0.91$. (For a similar correction from simulation see [96]).

On the other hand, the scale-dependent bias can be rewritten as

$$
b_{2 L}=\frac{I_{21}(k, m)}{2 \sigma_{m}^{2} M(k, z)} \delta_{c} b_{1 L}+\frac{1}{M(k, z)} \partial_{\ln \sigma_{m}^{2}}\left(\frac{I_{21}(k, m)}{\sigma_{m}^{2}}\right) .
$$

So the total Eulerian bias up to first order in $f_{N L}$ can be split to scale-independent $b_{s i}$ and scale-dependent $b_{s d}$ terms as

$$
b_{t}=b_{s i}+b_{s d}
$$

where $b_{s i}$ and $b_{s d}$ are

$$
b_{s i} \equiv b_{G}+b_{1 L(N G)}
$$

with $b_{G} \equiv 1+b_{1 L(G)}$ and

$$
b_{s d}=b_{2 L}+\mathcal{O}\left(f_{N L}^{2}\right)+\mathcal{O}\left(g_{N L}\right)+\ldots
$$

[1] A. H. Guth, "The Inflationary Universe: A Possible Solution To The Horizon And Flatness Problems," Phys. Rev. D 23, $347(1981)$ K. Sato, "First Order Phase Transition of a Vacuum and Expansion of the Universe," Mon. Not. Roy. Astron. Soc. 195, 467-479 (1981).

A. D. Linde, "A New Inflationary Universe Scenario: A Possible Solution Of The Horizon, Flatness, Homogeneity, Isotropy And Primordial Monopole Problems," Phys. Lett. B 108, 389 (1982);

A. Albrecht and P. J. Steinhardt, "Cosmology For Grand Unified Theories With Radiatively Induced Symmetry Breaking," Phys. Rev. Lett. 48, 1220 (1982). 
[2] G. Hinshaw, D. Larson, E. Komatsu, D. N. Spergel, C. L. Bennett, J. Dunkley, M. R. Nolta and M. Halpern et al., "NineYear Wilkinson Microwave Anisotropy Probe (WMAP) Observations: Cosmological Parameter Results," arXiv:1212.5226 [astro-ph.CO].

[3] P. A. R. Ade et al. [Planck Collaboration], "Planck 2013 results. XVI. Cosmological parameters," arXiv:1303.5076] [astroph.CO].

[4] X. Chen, "Primordial Non-Gaussianities from Inflation Models," Adv. Astron. 2010, 638979 (2010) arXiv:1002.1416 [astro-ph.CO]].

[5] E. Komatsu, "Hunting for Primordial Non-Gaussianity in the Cosmic Microwave Background," Class. Quant. Grav. 27, 124010 (2010) arXiv:1003.6097 [astro-ph.CO]].

[6] N. Bartolo, S. Matarrese and A. Riotto, "Non-Gaussianity and the Cosmic Microwave Background Anisotropies," Adv. Astron. 2010, 157079 (2010) arXiv:1001.3957 [astro-ph.CO]].

[7] J. M. Maldacena, "Non-Gaussian features of primordial fluctuations in single field inflationary models," JHEP 0305, 013 (2003) astro-ph/0210603.

[8] M. H. Namjoo, H. Firouzjahi and M. Sasaki, "Violation of non-Gaussianity consistency relation in a single field inflationary model," arXiv:1210.3692 [astro-ph.CO].

[9] X. Chen, H. Firouzjahi, M. H. Namjoo and M. Sasaki, "A Single Field Inflation Model with Large Local Non-Gaussianity," arXiv:1301.5699 [hep-th].

[10] I. Agullo and L. Parker, "Non-gaussianities and the Stimulated creation of quanta in the inflationary universe," Phys. Rev. D 83, 063526 (2011) arXiv:1010.5766 [astro-ph.CO]].

[11] A. Ashoorioon and G. Shiu, "A Note on Calm Excited States of Inflation," JCAP 1103, 025 (2011) arXiv:1012.3392 [astro-ph.CO]].

[12] J. Ganc, "Calculating the local-type fNL for slow-roll inflation with a non-vacuum initial state," Phys. Rev. D 84, 063514 (2011) arXiv:1104.0244 [astro-ph.CO]].

[13] D. Chialva, "Signatures of very high energy physics in the squeezed limit of the bispectrum (violation of Maldacena's condition)," JCAP 1210, 037 (2012).

[14] P. A. R. Ade et al. [Planck Collaboration], "Planck 2013 Results. XXIV. Constraints on primordial non-Gaussianity," arXiv:1303.5084 [astro-ph.CO].

[15] E. Komatsu, B. D. Wandelt, D. N. Spergel, A. J. Banday and K. M. Gorski, "Measurement of the cosmic microwave background bispectrum on the COBE DMR sky maps," Astrophys. J. 566, 19 (2002) astro-ph/0107605.

[16] E. Komatsu, D. N. Spergel and B. D. Wandelt, "Measuring primordial non-Gaussianity in the cosmic microwave background," Astrophys. J. 634, 14 (2005) astro-ph/0305189.

[17] N. Dalal, O. Dore, D. Huterer and A. Shirokov, "The imprints of primordial non-gaussianities on large-scale structure: scale dependent bias and abundance of virialized objects," Phys. Rev. D 77, 123514 (2008) [arXiv:0710.4560 [astro-ph]].

[18] R. Scoccimarro, E. Sefusatti and M. Zaldarriaga, "Probing primordial non-Gaussianity with large - scale structure," Phys. Rev. D 69, 103513 (2004) astro-ph/0312286.

[19] V. Desjacques and U. Seljak, "Primordial non-Gaussianity in the large scale structure of the Universe," Adv. Astron. 2010, 908640 (2010) arXiv:1006.4763 [astro-ph.CO]].

[20] W. H. Press and P. Schechter, "Formation of galaxies and clusters of galaxies by selfsimilar gravitational condensation," Astrophys. J. 187, 425 (1974).

[21] L. Verde, "Non-Gaussianity from Large-Scale Structure Surveys," Adv. Astron. 2010, 768675 (2010) arXiv:1001.5217 [astro-ph.CO]].

[22] S. Matarrese, L. Verde and R. Jimenez, "The Abundance of high-redshift objects as a probe of non-Gaussian initial conditions," Astrophys. J. 541, 10 (2000) astro-ph/0001366.

[23] M. Kamionkowski, L. Verde and R. Jimenez, "The Void Abundance with Non-Gaussian Primordial Perturbations," JCAP 0901, 010 (2009) arXiv:0809.0506 [astro-ph]].

[24] M. LoVerde, A. Miller, S. Shandera and L. Verde, "Effects of Scale-Dependent Non-Gaussianity on Cosmological Structures," JCAP 0804, 014 (2008) [arXiv:0711.4126 [astro-ph]].

[25] M. Grossi, L. Verde, C. Carbone, K. Dolag, E. Branchini, F. Iannuzzi, S. Matarrese and L. Moscardini, "Large-scale non-Gaussian mass function and halo bias: tests on N-body simulations," Mon. Not. Roy. Astron. Soc. 398, 321 (2009) arXiv:0902.2013 [astro-ph.CO]].

[26] P. Catelan, F. Lucchin, S. Matarrese, and L. Moscardini. Eulerian perturbation theory in non-flat universes: second-order approximation. MNRAS, 276:3956, September 1995.

[27] D. Jeong and E. Komatsu, "Primordial non-Gaussianity, scale-dependent bias, and the bispectrum of galaxies," Astrophys. J. 703, 1230 (2009) arXiv:0904.0497 [astro-ph.CO]].

[28] T. Nishimichi, A. Taruya, K. Koyama and C. Sabiu, "Scale Dependence of Halo Bispectrum from Non-Gaussian Initial Conditions in Cosmological N-body Simulations," JCAP 1007, 002 (2010) arXiv:0911.4768] [astro-ph.CO]].

[29] T. Baldauf, U. Seljak and L. Senatore, "Primordial non-Gaussianity in the Bispectrum of the Halo Density Field," JCAP 1104, 006 (2011) arXiv:1011.1513 [astro-ph.CO]].

[30] S. Matarrese and L. Verde, "The effect of primordial non-Gaussianity on halo bias," Astrophys. J. 677, L77 (2008) arXiv:0801.4826 [astro-ph]].

[31] N. Afshordi and A. J. Tolley, "Primordial non-gaussianity, statistics of collapsed objects, and the Integrated Sachs-Wolfe effect," Phys. Rev. D 78, 123507 (2008) arXiv:0806.1046 [astro-ph]].

[32] B. A. Reid, L. Verde, K. Dolag, S. Matarrese and L. Moscardini, "Non-Gaussian halo assembly bias," JCAP 1007, 013 (2010) arXiv:1004.1637 [astro-ph.CO]]. 
[33] E. Sefusatti, M. Crocce and V. Desjacques, "The Halo Bispectrum in N-body Simulations with non-Gaussian Initial Conditions," arXiv:1111.6966 [astro-ph.CO].

[34] J. -Q. Xia, C. Baccigalupi, S. Matarrese, L. Verde and M. Viel, "Constraints on Primordial Non-Gaussianity from Large Scale Structure Probes," JCAP 1108, 033 (2011) arXiv:1104.5015 [astro-ph.CO]].

[35] A. R. Pullen and C. M. Hirata, "Systematic effects in large-scale angular power spectra of photometric quasars and implications for constraining primordial nongaussianity," arXiv:1212.4500 [astro-ph.CO].

[36] T. Giannantonio, A. J. Ross, W. J. Percival, R. Crittenden, D. Bacher, M. Kilbinger, R. Nichol and J. Weller, "Improved Primordial Non-Gaussianity Constraints from Measurements of Galaxy Clustering and the Integrated Sachs-Wolfe Effect," arXiv:1303.1349 [astro-ph.CO].

[37] T. G. Sarkar and D. K. Hazra, "Probing primordial non-Gaussianity: The 3D Bispectrum of Ly-alpha forest and the redshifted 21-cm signal from the post reionization epoch," arXiv:1211.4756 [astro-ph.CO].

[38] D. K. Hazra and T. G. Sarkar, "Primordial Non-Gaussianity in the Forest: 3D Bispectrum of Ly-alpha Flux Spectra Along Multiple Lines of Sight," Phys. Rev. Lett. 109, 149902 (2012) [arXiv:1205.2790 [astro-ph.CO]].

[39] G. D’Amico, M. Musso, J. Norena and A. Paranjape, "Excursion Sets and Non-Gaussian Void Statistics," Phys. Rev. D 83, 023521 (2011) arXiv:1011.1229 [astro-ph.CO]].

[40] C. Fedeli, M. Bartelmann and L. Moscardini, "Constraining primordial non-Gaussianity with cosmological weak lensing: shear and flexion," JCAP 1210, 018 (2012) arXiv:1204.2229 [astro-ph.CO]].

[41] D. Hanson, A. Lewis, "Estimators for CMB Statistical Anisotropy," Phys. Rev. D80, 063004 (2009). arXiv:0908.0963 [astro-ph.CO]].

[42] D. Hanson, A. Lewis, A. Challinor, "Asymmetric Beams and CMB Statistical Anisotropy," Phys. Rev. D81, 103003 (2010). arXiv:1003.0198 [astro-ph.CO]].

[43] P. A. R. Ade et al. [Planck Collaboration], "Planck 2013 results. XXIII. Isotropy and Statistics of the CMB," arXiv:1303.5083 [astro-ph.CO].

[44] L. Ackerman, S. M. Carroll and M. B. Wise, "Imprints of a Primordial Preferred Direction on the Microwave Background," Phys. Rev. D 75, 083502 (2007) [Erratum-ibid. D 80, 069901 (2009)] astro-ph/0701357.

[45] N. E. Groeneboom, L. Ackerman, I. K. Wehus and H. K. Eriksen, "Bayesian analysis of an anisotropic universe model: systematics and polarization," Astrophys. J. 722, 452 (2010) arXiv:0911.0150 [astro-ph.CO]].

[46] A. R. Pullen and C. M. Hirata, "Non-detection of a statistically anisotropic power spectrum in large-scale structure," JCAP 1005, 027 (2010) arXiv:1003.0673 [astro-ph.CO]].

[47] M. a. Watanabe, S. Kanno and J. Soda, "Inflationary Universe with Anisotropic Hair," Phys. Rev. Lett. 102, 191302 (2009) arXiv:0902.2833 [hep-th]].

[48] N. Bartolo, S. Matarrese, M. Peloso and A. Ricciardone, "The anisotropic power spectrum and bispectrum in the $f(\phi) F^{2}$ mechanism," arXiv:1210.3257 [astro-ph.CO].

[49] M. Shiraishi, E. Komatsu, M. Peloso and N. Barnaby, "Signatures of anisotropic sources in the squeezed-limit bispectrum of the cosmic microwave background," arXiv:1302.3056 [astro-ph.CO].

[50] A. A. Abolhasani, R. Emami, J. T. Firouzjaee and H. Firouzjahi, " $\delta N$ Formalism in Anisotropic Inflation and Large Anisotropic Bispectrum and Trispectrum," arXiv:1302.6986 [astro-ph.CO].

[51] D. H. Lyth and M. Karciauskas, "The statistically anisotropic curvature perturbation generated by $f(\phi)^{2} F^{2}, "$ arXiv:1302.7304 [astro-ph.CO].

[52] M. S. Turner and L. M. Widrow, "Inflation Produced, Large Scale Magnetic Fields," Phys. Rev. D 37, 2743 (1988).

[53] B. Ratra, "Cosmological 'seed' magnetic field from inflation," Astrophys. J. 391, L1 (1992).

[54] K. Yamamoto, "Primordial Fluctuations from Inflation with a Triad of Background Gauge Fields," Phys. Rev. D 85, 123504 (2012) arXiv:1203.1071 [astro-ph.CO]].

[55] R. Emami and H. Firouzjahi, "Curvature Perturbations in Anisotropic Inflation with Symmetry Breaking," arXiv:1301.1219 [hep-th].

[56] T. R. Dulaney, M. I. Gresham, "Primordial Power Spectra from Anisotropic Inflation," Phys. Rev. D81, 103532 (2010). arXiv:1001.2301 [astro-ph.CO]].

[57] A. E. Gumrukcuoglu, B. Himmetoglu, M. Peloso, "Scalar-Scalar, Scalar-Tensor, and Tensor-Tensor Correlators from Anisotropic Inflation," Phys. Rev. D81, 063528 (2010). arXiv:1001.4088 [astro-ph.CO]].

[58] M. a. Watanabe, S. Kanno and J. Soda, "The Nature of Primordial Fluctuations from Anisotropic Inflation," Prog. Theor. Phys. 123, 1041 (2010) arXiv:1003.0056 [astro-ph.CO]].

[59] H. Funakoshi and K. Yamamoto, "Primordial bispectrum from inflation with background gauge fields," arXiv:1212.2615 [astro-ph.CO].

[60] A. R. Pullen and M. Kamionkowski, "Cosmic Microwave Background Statistics for a Direction-Dependent Primordial Power Spectrum," Phys. Rev. D 76, 103529 (2007) arXiv:0709.1144 [astro-ph]].

[61] R. Scoccimarro, L. Hui, M. Manera and K. C. Chan, "Large-scale Bias and Efficient Generation of Initial Conditions for Non-Local Primordial Non-Gaussianity," Phys. Rev. D 85, 083002 (2012) arXiv:1108.5512 [astro-ph.CO]].

[62] P. Adshead, E. J. Baxter, S. Dodelson and A. Lidz, "Non-Gaussianity and Excursion Set Theory: Halo Bias," Phys. Rev. D 86, 063526 (2012) arXiv:1206.3306 [astro-ph.CO]].

[63] A. D'Aloisio, J. Zhang, D. Jeong and P. R. Shapiro, "Halo statistics in non-Gaussian cosmologies: the collapsed fraction, conditional mass function, and halo bias from the path-integral excursion set method," Mon. Not. Roy. Astron. Soc. 428, 2765 (2013) arXiv:1206.3305 [astro-ph.CO]].

[64] J.R. Bond, S. Cole, G. Efstathiou and N. Kaiser, Astrophys. J. 379, 440 (1991).

[65] R. K. Sheth and G. Lemson, "Biasing and the distribution of dark matter haloes," astro-ph/9808138. 
[66] H. J. Mo, Y. P. Jing and S. D. M. White, "High-order correlations of peaks and halos: A Step toward understanding galaxy biasing," astro-ph/9603039.

[67] R. Scoccimarro, R. K. Sheth, L. Hui and B. Jain, "How many galaxies fit in a halo? Constraints on galaxy formation efficiency from spatial clustering," Astrophys. J. 546, 20 (2001) astro-ph/0006319.

[68] R. E. Smith, R. Scoccimarro and R. K. Sheth, "The Scale Dependence of Halo and Galaxy Bias: Effects in Real Space," Phys. Rev. D 75, 063512 (2007) astro-ph/0609547.

[69] M. Manera, R. KSheth and R. Scoccimarro, "Large scale bias and the inaccuracy of the peak-background split," arXiv:0906.1314 [astro-ph.CO].

[70] M. Manera and E. Gaztanaga, "The local bias model in the large scale halo distribution," arXiv:0912.0446] [astro-ph.CO].

[71] J. E. Gunn and J. R. Gott, III, "On the Infall of Matter into Clusters of Galaxies and Some Effects on Their Evolution," Astrophys. J. 176, 1 (1972).

[72] S. Cole and N. Kaiser, Mon. Not. R. Astron. Soc. 237, 1127 (1989)

[73] J. M. Bardeen, J. R. Bond, N. Kaiser and A. S. Szalay, "The Statistics of Peaks of Gaussian Random Fields," Astrophys. J. 304, 15 (1986).

[74] A. Slosar, C. Hirata, U. Seljak, S. Ho and N. Padmanabhan, JCAP 68, 031 (2008).

[75] M. Maggiore and A. Riotto, "The Halo mass function from excursion set theory. III. Non-Gaussian fluctuations," Astrophys. J. 717, 526 (2010) arXiv:0903.1251 [astro-ph.CO]].

[76] M. Maggiore and A. Riotto, "The Halo Mass Function from Excursion Set Theory. I. Gaussian fluctuations with nonMarkovian dependence on the smoothing scale," Astrophys. J. 711, 907 (2010) [arXiv:0903.1249 [astro-ph.CO]].

[77] M. Maggiore and A. Riotto, "The Halo mass function from excursion set theory. II. The diffusing barrier," Astrophys. J. 717, 515 (2010) arXiv:0903.1250 [astro-ph.CO]].

[78] A. Paranjape and R. K. Sheth, "Halo bias in the excursion set approach with correlated steps," Mon. Not. Roy. Astron. Soc. 419 (2012) 132 arXiv:1105.2261 [astro-ph.CO]].

[79] A. Paranjape, T. Y. Lam and R. K. Sheth, "Halo abundances and counts-in-cells: The excursion set approach with correlated steps," Mon. Not. Roy. Astron. Soc. 420, 1429 (2012) arXiv:1105.1990 [astro-ph.CO]].

[80] M. Musso and A. Paranjape, "Non-Gaussian halo abundances in the excursion set approach with correlated steps," Mon. Not. Roy. Astron. Soc. 420, 369 (2012) arXiv:1108.0565 [astro-ph.CO]].

[81] M. Musso and R. K. Sheth, "One step beyond: The excursion set approach with correlated steps," Mon. Not. Roy. Astron. Soc. 423, L102 (2012) arXiv:1201.3876 [astro-ph.CO]].

[82] M. Musso, A. Paranjape and R. K. Sheth, "Scale dependent halo bias in the excursion set approach," Mon. Not. Roy. Astron. Soc. 427, 3145 (2012) [arXiv:1205.3401] [astro-ph.CO]].

[83] H. J. Mo and S. D. M. White, "An Analytic model for the spatial clustering of dark matter halos," Mon. Not. Roy. Astron. Soc. 282, 347 (1996) astro-ph/9512127.

[84] P. Catelan, F. Lucchin, S. Matarrese and C. Porciani, "The bias field of dark matter halos," Mon. Not. Roy. Astron. Soc. 297, 692 (1998) astro-ph/9708067.

[85] G. Efstathiou, C. S. Frenk, S. D. M. White and M. Davis, "Gravitational clustering from scale free initial conditions," Mon. Not. Roy. Astron. Soc. 235, 715 (1988).

[86] S. Cole and N. Kaiser, "Biased clustering in the cold dark matter cosmogony," Mon. Not. Roy. Astron. Soc. 237, 1127 (1989).

[87] E. Sefusatti, C. Vale, K. Kadota and J. Frieman, "Primordial non-Gaussianity and Dark Energy constraints from Cluster Surveys," Astrophys. J. 658, 669 (2007) astro-ph/0609124.

[88] R. K. Sheth and G. Tormen, "Large scale bias and the peak background split," Mon. Not. Roy. Astron. Soc. 308, 119 (1999) astro-ph/9901122.

[89] M. Shiraishi, S. Yokoyama, K. Ichiki and T. Matsubara, "Scale-dependent bias due to primordial vector field," arXiv:1301.2778 [astro-ph.CO].

[90] E. Sefusatti, J. R. Fergusson, X. Chen and E. P. S. Shellard, "Effects and Detectability of Quasi-Single Field Inflation in the Large-Scale Structure and Cosmic Microwave Background," JCAP 1208, 033 (2012) [arXiv:1204.6318 [astro-ph.CO]].

[91] S. Weinberg, Cosmology, Oxford University Press, (2008).

[92] D. S. Salopek and J. R. Bond, "Nonlinear evolution of long wavelength metric fluctuations in inflationary models," Phys. Rev. D 42 (1990) 3936.

[93] E. Komatsu and D. N. Spergel, "Acoustic signatures in the primary microwave background bispectrum," Phys. Rev. D 63, 063002 (2001) astro-ph/0005036.

[94] A. Paranjape, C. Gordon and S. Hotchkiss, "The Extreme Tail of the Non-Gaussian Mass Function," Phys. Rev. D 84, 023517 (2011) arXiv:1104.1145 [astro-ph.CO]].

[95] V. Desjacques, U. Seljak and I. Iliev, "Scale-dependent bias induced by local non-Gaussianity: A comparison to N-body simulations," arXiv:0811.2748 [astro-ph].

[96] J. L. Tinker, A. V. Kravtsov, A. Klypin, K. Abazajian, M. S. Warren, G. Yepes, S. Gottlober and D. E. Holz, "Toward a halo mass function for precision cosmology: The Limits of universality," Astrophys. J. 688, 709 (2008) arXiv:0803.2706 [astro-ph]].

[97] H. Zhan, "Cosmic tomographies: baryon acoustic oscillations and weak lensing," JCAP 0608, 008 (2006) astro-ph/0605696.

[98] A. D'Aloisio, J. Zhang, P. R. Shapiro and Y. Mao, "The scale-dependent signature of primordial non-Gaussianity in the large-scale structure of cosmic reionization," arXiv:1304.6411 [astro-ph.CO].

[99] A. Lidz, E. J. Baxter, P. Adshead and S. Dodelson, "Primordial Non-Gaussianity and Reionization," arXiv:1304.8049 
[astro-ph.CO].

[100] S. Camera, M. G. Santos, P. G. Ferreira and L. Ferramacho, "Cosmology on Ultra-Large Scales with HI Intensity Mapping: Limits on Primordial non-Gaussianity," arXiv:1305.6928 [astro-ph.CO].

[101] Y. Mao, A. D'Aloisio, J. Zhang and P. R. Shapiro, "Primordial Non-Gaussianity Estimation using $21 \mathrm{~cm}$ Tomography from the Epoch of Reionization," arXiv:1305.0313 [astro-ph.CO]. 